\title{
The effect of spatiotemporal antibiotic inhomogeneities on the evolution of resistance
}

\author{
Harrison Steel and Antonis Papachristodoulou \\ Authors are with the Department of Engineering Science, University of Oxford, Oxford, OX1 3PJ, \\ UK. Corresponding Author: Harrison Steel, harrison.steel@eng.ox.ac.uk.
}

\begin{abstract}
Combating the evolution of widespread antibiotic resistance is one of the most pressing challenges facing modern medicine. Recent research has demonstrated that the evolution of pathogens with high levels of resistance can be accelerated by spatial and temporal inhomogeneities in antibiotic concentration, which frequently arise in patients and the environment. Strategies to predict and counteract the effects of such inhomogeneities will be critical in the fight against resistance. In this paper we develop a mechanistic framework for modelling the adaptive evolution of resistance in the presence of spatiotemporal antibiotic concentrations, which treats the adaptive process as an interaction between two mutually orthogonal forces; the first returns cells to their wild-type state in the absence of antibiotic selection, and the second selects for increased coping ability in the presence of an antibiotic. We apply our model to investigate laboratory adaptation experiments, and then extend it to consider the case in which multiple strategies for resistance undergo competitive evolution.
\end{abstract}

Keywords:

Antibiotic resistance; Evolution; Spatio-temporal drug gradients; Evolutionary models

\section{Introduction}

Combatting the evolution of antibiotic resistant pathogens is one of the major clinical challenges faced by modern medicine (Ling et al., 2015; Durão et al., 2018; Baym et al., 2016b). Given sufficient time, resistance to virtually all antibiotic compounds can evolve (Bell and MacLean, 2018), and because resistant strains tend to adapt themselves to their new conditions (MacLean et al., 2010; Andersson, 2006) the return to sensitivity (in the absence of an antibiotic) occurs very slowly (Durão et al., 2018; Schrag et al., 1997; De Gelder et al., 2004). Consequently there is a pressing need for new approaches to predicting and preventing the spread of resistance, and its evolution in the first place (Gifford et al., 2018; Furusawa et al., 2018). This will require novel theoretical descriptions of the development of resistance (Bell and MacLean, 2018; Lukačišinová and Bollenbach, 2017) which take into account a range of environmental and physical factors that regulate the process. If these techniques can be employed to sufficiently slow down the development of resistance, its spread may eventually be stopped entirely (Bell and MacLean, 2018).

Cell-Antibiotic interactions vary greatly depending on the particular species involved: Antibiotics may target a broad range of cellular processes and systems, and can have widely varying effects on different cell lines or species. These antibiotic effects can be broadly classified as bactericidal 
(killing of bacteria) or bacteriostatic (preventing of growth) (Kohanski et al., 2010). Antibiotic effects are typically quantified using metrics such as the MBC (minimum bactericidal concentration) or MIC (minimum inhibitory concentration) which represent concentrations required to almost entirely eliminate a bacterial population (Liu et al., 2004; Wang et al., 2016). However, these metrics can be difficult to apply (particularly in clinical settings) due to the wide range of responses cells may have to high antibiotic concentrations. For example, cells can exhibit tolerant (reduced or stalled growth which allows antibiotics that require active growth for killing to be resisted) or persister (non-growing clonal sub-populations) behaviours which allow populations to recover following prolonged antibiotic exposure (Brauner et al., 2016; Harms et al., 2016; Cohen et al., 2013).

At the biochemical level antibiotic resistance can be achieved by a range of mechanisms, depending on the environmental conditions and antibiotic in question (Palmer et al., 2018; Pál et al., 2015). These can be broadly classified into four mechanistic categories: The modification of cellular targets so that antibiotic binding is diminished, physical removal of an antibiotic from the cell via modification of efflux systems, reduction of cellular uptake, and enzymatic inactivation of the antibiotic (Pál et al., 2015). When one of these forms of resistance develops via a series of mutations there are, in many cases, only a limited number of evolutionary pathways that can be taken (Weinreich et al., 2006; Palmer et al., 2015). Evolutionary trajectories have proven to be remarkably reproducible (Weinreich et al., 2006; Chevereau et al., 2015; Didelot et al., 2016), and the particular trajectory taken can be influenced by a range of environmental conditions (Gifford et al., 2018; Lukačišinová and Bollenbach, 2017; Zampieri et al., 2017). Similar evolutionary outcomes often have very different effects on other cellular behaviours, such as cross-sensitivity to other antibiotics (Barbosa et al., 2017).

Recent studies have demonstrated that the acquisition of resistance can be significantly influenced by spatial (Zhang et al., 2011; Baym et al., 2016a) or temporal (Toprak et al., 2012; Lindsey et al., 2013; Chevereau et al., 2015; Oz et al., 2014; Zampieri et al., 2017) inhomogeneities in antibiotic concentration. Such inhomogeneities have been shown to arise in both clinical settings (e.g. concentration differences between organs within a patient), as well as in external environments (such as rivers and lakes where antibiotic run-off accumulates) (Andersson and Hughes, 2014). The presence of intermediate antibiotic concentrations allows pathogens to gradually develop greater levels of resistance, making them more able to adapt to large antibiotic concentrations that are subsequently encountered (Bell and MacLean, 2018). During these adaptive processes resistance can increase continuously (e.g. when the expression of many genes is optimised) or in a step-wise manner (e.g. when a small number of mutational changes are responsible for determining overall resistance efficacy) (Toprak et al., 2012; Chevereau et al., 2015; Barrick and Lenski, 2013; Palmer et al., 2018). A particularly extreme case of step-wise resistance improvement is that due to horizontal transfer of genetic information (such as plasmids) that encode resistance genes (Jansen et al., 2013).

A range of mathematical models have been developed to describe the evolution and proliferation of antibiotic resistance (Opatowski et al., 2011; Mozhayskiy and Tagkopoulos, 2013). Competition and transmission models have proven to be valuable for modelling the spread of resistance within cellular populations, patients, or hospital and human populations at large (Spicknall et al., 2013; Jacobs et al., 2016). At the genetic level models of individual base-pair substitutions in DNA can describe adaptive processes over short time-scales (Posada and Crandall, 2001; Hindré et al., 2012), 
and analyse distributions of mutational effects (MacLean et al., 2010; Gillespie, 1984). Studies of mutational landscapes have demonstrated that these individual genetic mutations may combine to determine a particular trait in highly nontrivial ways (Furusawa et al., 2018; Beerenwinkel et al., 2007; Franke et al., 2011; Palmer et al., 2015). Traditional descriptions of long-term adaptive evolution have generally considered evolutionary pathways at a greater level of abstraction, modelling the variation in quantitative traits as random walk processes (Lande, 1976; Hansen, 1997; Beaulieu et al., 2012; Uyeda and Harmon, 2014). In a few cases stochastic models of evolution have been combined with consideration of spatial inhomogeneities (which models have shown impact genetic diversity (Behrman and Kirkpatrick, 2011) and the evolution of resistance (Kepler and Perelson, 1998; De Jong and Wood, 2018)) for the case of antibiotic resistance: Hermsen et al. proposed a staircase model which discretises bacteria in both space and phenotype, demonstrating that spatial antibiotic variation can encourage development of resistance (Hermsen et al., 2012). This was later developed to provide a continuum treatment of space and phenotype (Hermsen, 2016). Greulich et al. proposed a similar model (they are compared in (Hermsen, 2016)), which demonstrated that environmental inhomogeneities can slow the acquisition of resistance in some cases (Greulich et al., 2012). Finally, Gralka et al modelled the impact of convection in the presence of spatial inhomogeneities, examining the trade-off between antibiotic efficacy and the potential for resistance to arise (Gralka et al., 2017).

Building upon this past work, in this paper we outline a mathematical framework for describing the evolution of resistance in the presence of spatiotemporal antibiotic inhomogeneities. We investigate how different aspects of the evolutionary process determine the rate at which resistance emerges, and demonstrate how our framework can be used to analyse the results of past studies and common clinical situations.

\section{Methods}

\subsection{A Cell's Coping Ability}

To model the evolution of antibiotic resistance we propose a parameterisation of cell fitness, coping ability $(\gamma)$, which reflects the ability of a particular antibiotic resistance strategy to permit a cell to reproduce when stressed by a coping challenge $\left(\gamma_{c}\right)$ : If a given cell has $\gamma<\gamma_{c}$ (noting that $\gamma_{c}$ may be location or time dependent) then it is unable to reproduce. By parameterising resistance in this way we assume that $\gamma$ is a function of both genetic changes, as well as any other heritable phenotypic differences between cells that impact their interactions with an antibiotic (Deris et al., 2013; Erickson et al., 2016). Many resistance strategies only arise when mutations are present in certain combinations (Weinreich et al., 2006), which motivates a parameterisation of resistance $(\gamma)$ which is not reducible to a sum of individual effects (i.e. discrete point mutations), since any such reduction would require assumptions be made about all potential combinatorial effects of individual mutations.

The coping challenge $\left(\gamma_{c}\right)$ is assumed to be a non-decreasing function of local antibiotic concentration, and may be non-linear. The functional dependence of $\gamma_{c}$ upon antibiotic concentration is likely to differ between antibiotics depending on their mechanism of action. However, one potential relationship is of the form:

$$
\gamma_{c}=\log \left([A] /[A]_{50}\right)
$$


where $[A]$ is antibiotic concentration and $[A]_{50}$ is the antibiotic concentration required to prevent growth of $50 \%$ of wild-type cells (often referred to as $\mathrm{IC}_{50}$ (Soothill et al., 1992)). When no antibiotic is present $([A]=0)$ we have $\gamma_{c}=-\infty$, and thus any value of $\gamma$ is sufficient for all cells to reproduce. Eq. (1) aligns with the results of many experimental studies that have demonstrated that the challenge posed by adapting to an increase in antibiotic concentration is roughly proportional to the fold-change that must be overcome (Baym et al., 2016a; Pennell et al., 2015; Toprak et al., 2012).

A significant obstacle to formulating a general theory for adaptive evolution is the unknown distribution of fitness effects (Rokyta et al., 2005). For antibiotic resistance this metric is difficult to estimate because the beneficial (antibiotic resisting) effects of many mutations are outweighed by their fitness cost (MacLean et al., 2010). To side-step this challenge we decompose the evolutionary process into two orthogonal components. The first is the antibiotic $\left(\gamma_{c}\right)$ dependent reproduction mentioned above. The second determines the mutational dynamics of $\gamma$ in the absence of an antibiotic, and is governed by two processes:

Random Mutation $\left(D_{\gamma}\right)$ : Mutational variations in $\gamma$ are introduced over time as normally distributed increments (as observed in the distribution of fitness effects for non-optimal traits (Schenk et al., 2012; McDonald et al., 2011)) with zero mean, and thus have a 50\% probability of increasing $\gamma$ (but do not affect the cell's typical reproduction rate or longevity). Treatment of $\gamma$ as a continuous parameter is motivated by the observation that resistance is determined by the cumulative effect of many mutations that may impact diverse processes (Lukačišinová and Bollenbach, 2017; Feng et al., 2016), which often leads to a population's resistance developing continuously in time (Toprak et al., 2012).

Mean Reversion ( $\theta$ ): Mean reversion (drift in $\gamma$ toward its mean wild-type value $\mu$ ) is driven by the fitness cost that mutations impart upon a host cell when considered in the absence of an antibiotic. Long term studies have shown that this rate of reversion can vary significantly depending on the strain, drug, and resistance mechanism in question (Bean et al., 2005). To parameterise this process we assume that a mutational state's fitness cost is independent of the degree to which that state can resist the antibiotic, and as such mean reversion occurs at a constant rate $\theta$. All mutations therefore result in some degree of fitness loss (as they steer the cell away from its optimal wild-type state) when considered independently of the effect of antibiotic-driven selection. A constant ( $\gamma$-independent) reversion rate represents a minimal null-hypothesis when this dependence is unknown, and is supported by two experimental observations which provide mechanisms for decoupling the potency of resistance mutations from their fitness cost. First, organisms evolve to largely negate the fitness cost of resistance (MacLean et al., 2010; Andersson, 2006), often on a timescale that is faster than the development of resistance itself (Moura De Sousa et al., 2015). Second, a particular $\gamma$ value may result from a small number of mutations with large effect (or a large number with small effect) on resistance (Bloom and Arnold, 2009), and so in general larger $\gamma$ values do not necessarily incur a greater fitness cost.

We combine the factors above to model the dynamics of $\gamma$ in the absence of selection (i.e. zero antibiotic, $\left.\gamma_{c}=-\infty\right)$ with a stochastic differential equation of the form:

$$
\mathrm{d} \gamma_{t}=-\theta \operatorname{sign}\left(\gamma_{t}-\mu\right) \mathrm{d} t+\sqrt{2 D_{\gamma}} \mathrm{d} B_{t}
$$


Here $B_{t}$ is a Wiener process (Brownian motion), scaled by diffusion constant $D_{\gamma}$. Eq. (2) is an example of a stochastic differential equation (SDE) with discontinuous drift, for which the stationary solution is a Laplace distribution with $\mathbb{E}[\gamma]=\mu$ and $\operatorname{Var}[\gamma]=2 D_{\gamma}^{2} / \theta^{2}$ (Simonsen et al., ${ }^{175}$ 2013). The stationary distribution of coping ability will be (as highlighted by past studies of evolutionary processes (Bloom et al., 2007; Amitai et al., 2007)) an important determinant of a cell's ability to adapt when selection for this trait occurs (i.e. an antibiotic is encountered). We define $\phi=\sqrt{\operatorname{Var}[\gamma]}=\sqrt{2} D_{\gamma} / \theta$ as a convenient measure of variability for our system. We will also generally set $\mu=0$, such that $\mathbb{E}[\gamma]=0$ and a coping challenge $\gamma_{c}=0$ (corresponding to $[A]_{50}$ ) will prevent $50 \%$ of cells from replicating. A particle whose motion is described by Eq. (2) has a char-

acteristic speed $\propto \sqrt{D_{\gamma}}$ and distance $\propto \phi$, meaning that the characteristic timescale over which it traverses its stationary distribution is $t_{c} \propto \sqrt{D_{\gamma}} / \theta$. In Fig. 1 we illustrate the interplay between the distribution of $\gamma$ values for a population of cells and a temporal or spatial step in the value of $\gamma_{c}$.

Past studies of evolutionary processes have similarly employed stochastic differential equations to model the drift in trait values over time (Lande, 1976; Hansen, 1997). In some cases these treatments include pure Brownian motion (Hermsen, 2016), but without mean reversion the variance of a trait value grows linearly in time. Others have described adaptive evolution using an Ornstein-Uhlenbeck process (Hansen, 1997), in which the rate of mean-reversion is proportional to the distance of a trait from an optimal value $\mu^{*}$. Though this yields a bounded variance, it assumes that the drift process (toward $\mu^{*}$ ) is driven by selection for improvement in the trait being studied. The interpretation of the SDE in Eq. 2 differs markedly from past modelling philosophies: Here we have separated out the selection process which drives change in $\gamma$ in response to environmental pressures (which is instead imposed by $\gamma_{c}$-dependent reproduction) from the underlying mutation process that governs variation in $\gamma$ in the absence of antibiotic-driven selection (which is entirely described by Eq. (2)). In Note S7 we describe a discretised version of Eq. (2), which constrains $\gamma$ to only take integer multiples of a discretisation parameter $\omega$. This reflects the outcome of experimental studies which have found that in some cases resistance can develop in discontinuous jumps due to distinct high-impact mutations (Toprak et al., 2012; Chevereau et al., 2015; Barrick and Lenski, 2013), rather than as a continuous process.

\subsection{Spatio-Temporal Dynamics and Selection}

Each cell's (unconstrained) spatial diffusion is described by the stochastic differential equation:

$$
\mathrm{d} x_{t}=\sqrt{2 D_{x}} \mathrm{~d} B_{t}
$$

where $B_{t}$ is a Wiener process (Brownian motion) and $D_{x}$ is the spatial diffusion rate. The probability density function of the position of a particle whose motion is described by Eq. (3) is a normal distribution with $\mathbb{E}\left[x_{t}\right]=0$ and $\operatorname{Var}\left[x_{t}\right]=2 D_{x} t$ meaning that (given sufficient time) unconstrained cells will reach any given spatial location.

Each cell is assumed to die at rate $\delta$, and as such when $\gamma<\gamma_{c}$ the number of surviving cells in ${ }^{210}$ a population will decrease exponentially over time (as is observed experimentally (Brauner et al., 2017)). The reproduction rate of a cell $i$ is given by:

$$
R_{i}=(1+\delta)\left(1-\sum_{j} \frac{\Gamma\left(x_{i}-x_{j} \mid \sigma\right)}{k}\right) H\left(\gamma_{i}-\gamma_{c}\left(x_{i}, t\right)\right)
$$


where the $1+\delta$ pre-factor is a scaling factor of the reproduction rate that arises in non-dimensionalisation (see Note $\mathrm{S} 1$ ), and $H$ is the Heaviside step function. $\Gamma(\Delta x \mid \sigma)$ is a Gaussian interaction kernel that is convolved across all cell locations $x_{j}$ to calculate the local cell density (and hence resource availability) at $x_{i}$, which has interaction range (standard deviation) $\sigma$. Finally, $k$ is the spatial carrying capacity (resource availability) per unit distance, and $\gamma_{c}\left(x_{i}, t\right)$ is the coping challenge as a function of position and time. When a cell replicates a daughter cell with the same $\gamma$ is placed at the same location. For spatial simulations we will generally choose a $\gamma_{c}$ that is independent of time. However, if we assume every cell has the same location (equivalent to setting $\sigma \rightarrow \infty$ ) then our modelling approach can be used to analyse situations in which adaptation is driven by temporal (rather than spatial) variations in antibiotic concentration (i.e. $\gamma_{c}(t)$ ).

Considering Eq. (2) alongside the selective reproduction enforced by Eq. (4) allows our modelling framework to be compared to traditional mathematical descriptions of trait evolution. Models that use the Ornstein-Uhlenbeck process and its derivatives employ a single SDE that combines the impact of random mutational changes and selective pressure on a particular trait's value (Hansen, 1997; Beaulieu et al., 2012; Blomberg, 2012). However, in the framework proposed herein these two factors enter as two independent selective forces: First, selection due to an antibiotic challenge $\gamma_{c}$ is imposed by selective reproduction following from Eq. (4). Second, a restoring force arising from the fitness cost of resistance mutations (in the absence of antibiotic) is imposed by the drift term (with rate $\theta$ ) in Eq. (2).

\subsection{Model Implementation}

For many situations that we wish to analyse our model will be analytically intractable, necessitating analysis via numerical simulation. To achieve this the model was implemented in MATLAB (template code is provided with the supplementary material) and numerically integrated as described in Note S2. The parameter values described in Note S3 are used unless otherwise specified in the Supplementary Notes that accompany each result. Though our model considers the impact of local resource competition between cells, it does not explicitly model the consumption of a finite resource pool. To account for this simulations are run for a finite amount of time, and the maximum time permitted is either interpreted as a literal time period, or a limitation imposed by an eventual exhaustion of resources. In simulations and subsequent figures we often express $\gamma$ values in multiples of $\phi$ (the standard deviation of the steady-state $\gamma$ distribution in the absence of antibiotic) to enable direct comparison between different simulated scenarios.

\subsection{Modelling Multiple Resistance Strategies}

We consider a situation in which our cell's total coping ability $\bar{\gamma}$ is determined by the combination of multiple mutually orthogonal adaptation strategies, $\gamma_{i}$, each governed by Eq. (2). The mutual orthogonality of these strategies implies that the correlation between their Brownian motion terms (as in Eq. (2)) is zero (or at least very small), and that there is no functional dependence between their mutation processes (i.e. $D_{\gamma_{i}}$ is not a function of $\gamma_{j}$ for $i \neq j$ ). Examples of orthogonal strategies are those for which a change in the effectiveness of one strategy does not directly affect the potential for improvement in the other. For example, if $\gamma_{1}$ represents adaptation primarily driven by development of efflux systems, and $\gamma_{2}$ represents adaptation primarily driven by active-site mutation, then we anticipate that mutations which cause a substantial increase in $\gamma_{1}$ will not necessarily impact $\gamma_{2}$ (though they may impact $\bar{\gamma}$ ). An example of two non-orthogonal strategies would be if we chose $\gamma_{1,2}$ to both represent different paths of active site mutation, and 
thus improvement in one could force improvement in the other (e.g. correlated Brownian motion), or the state of one path could influence the rate of change of the other (e.g. if they can be interconverted via a small number of mutations (Palmer et al., 2015)). For $\bar{\gamma}$ we propose a function of the form:

$$
\bar{\gamma}=\sum \gamma_{i}
$$

which is motivated by the following assumptions: When strategies combine to determine $\bar{\gamma}$ they should do so multiplicatively in terms of antibiotic concentration, and hence additively in terms of $\gamma$ (following from Eq. (1)). This means that if (for example) $\gamma_{i}$ represents an efflux pump strategy which removes $90 \%$ of an antibiotic from the cell's interior, then each $\gamma_{j}(j \neq i)$ evolves as if it is subjected to $10 \%$ of the total antibiotic concentration. When a new strategy is considered (i.e. adding an additional $\gamma_{i}$ to the calculation in Eq. (5)) the value of $D_{\gamma_{i}}$ will depend on how readily this strategy can evolve to impact $\bar{\gamma}$ (if $\gamma_{i}$ has minimal impact on a cell's coping ability then $\left.D_{\gamma_{i}} \approx 0\right)$. Consequently we set each $\mu_{i}=0$ such that considering a new strategy in Eq. (5) will not impact $\bar{\gamma}$ (unless it has evolved to contribute some form of resistance). Because each strategy is governed by an independent Wiener process in Eq. (2) we have (when at steady state with $\left.\gamma_{c}=-\infty\right)$ that $\bar{\phi}^{2}=\operatorname{Var}[\bar{\gamma}]=\sum \phi_{i}^{2}$.

\section{Results}

3.1. Adaptation driven by spatial variation in antibiotic concentration

A range of experimental and theoretical studies have demonstrated that spatial gradients (Zhang et al., 2011) or steps (Baym et al., 2016a) in antibiotic concentration can accelerate the acquisition of resistance conferring mutations in bacterial populations. In Fig. 2 we examine the adaptive behaviour predicted by our model to explain these results.

Fig. 2a illustrates a population of cells that encounters a spatial ramp in antibiotic concentration. Near $x \approx 0, \gamma_{c}$ is well below the wild-type cell's resilience, and so an approximately Laplacian distribution of coping abilities is maintained. As $\gamma_{c}$ rises it imparts a selection bias upon the population, favouring cells with larger $\gamma$ and driving up the mean coping ability. At the leading edge of the adapting front the variability of $\gamma$ values is substantially decreased because cells that migrate into free spatial regions can rapidly reproduce (due to the lack of resource competition), meaning that colonisation is driven by cells with $\gamma$ values near to the mean (local) value (and thus the evolutionary process is not driven by only its fittest constituents (Baym et al., 2016a)). There is an optimal antibiotic gradient at which $\gamma$ grows most quickly (Fig. 2b), originally investigated (using a model that is for this case similar to ours) by Hermsen (Hermsen, 2016). In Fig. S1 we provide examples similar to Fig. 2a that illustrate this behaviour for different gradients: When $\alpha<<1$ adaptation is limited by the rate of spatial diffusion $\left(D_{x}\right)$, whereas for $\alpha>>1$ adaptation is limited by rate at which variation in coping ability occurs $\left(D_{\gamma}\right)$ (Hermsen, 2016). In the large $\alpha$ case variability in $\gamma$ decreases, with the population's average gathering near its optimum (as found in previous studies of population evolution in changing environments (Droz and Pkalski, 2006)), which impedes adaptation since cells at the adaptive front mix extensively with those with lower $\gamma$. This phenomenon has been experimentally observed to slow adaptation at range margins (Bridle and Vines, 2007). In Fig. 2b the observed increase in adaptation rate at greater population densities $(k)$ aligns with the experimental observation that the rate at which resistance develops is increased for larger inoculum sizes (Gould and MacKenzie, 2002). 
Fig. 2c illustrates the response of a population of cells to a series of steps in antibiotic concentration: The first step is overcome, but within the given time the population is unable to make the subsequent jump to the region of higher concentration. We find that the probability of overcoming a fixed step in antibiotic is near zero for small population densities, but rises to unity when the cell density increases (Fig. 2d). This mirrors the experimental observations of Hol et al., who found that a critical bacterial density must be overcome in order to overcome a spatial step in antibiotic concentration (Hol et al., 2016). In Fig. 2e we simulate a colony of cells as it attempts to colonise a region of large antibiotic concentration by first colonising a step of intermediate height (the situation illustrated in Fig. 2c). The rate of colonisation is maximal when the intermediate step is approximately half of the final step size, which aligns with the experimental results of Baym et al. (Baym et al., 2016a). In Fig. 2f we model the interaction of our system with a mutagen which scales cell-wide mutation rates by a factor $\epsilon$ (see Note S12 for full description). This reveals an optimal range of mutation rates within which a population has a high probability of overcoming a step in antibiotic concentration: For small mutation rates variability in $\gamma$ is too small to overcome the antibiotic step, whilst for high mutation rates accelerated cell death reduces cell density and hence their ability to adapt. This simulation parallels an experimental study by Gerrish et al. (Gerrish and García-Lerma, 2003), where it was observed that both low and high mutation rates reduced the ability of a pathogen to adapt to a drug treatment.

\subsection{Adaptation driven by temporal variation in antibiotic concentration}

As with spatial gradients, experimental studies have demonstrated that temporal gradients and steps in antibiotic concentration can accelerate the evolution of resistant cell lines (Toprak et al., 2012; Lindsey et al., 2013; Chevereau et al., 2015; Oz et al., 2014; Zampieri et al., 2017). We now adjust our model (for details see Note S3) to consider a single, well mixed population (as might be found in a shaken liquid media experiment) for which spatial organisation of cells is not important.

When presented with a temporal antibiotic ramp $\left(\gamma_{c}=\alpha t\right)$ the mean $\gamma$ value for a cell population approximately coincides with $\gamma_{c}$ (Fig. 3a). For large $\alpha$ the cells are no longer able to mutate sufficiently quickly, and we observe a rapid drop in population density and survivability (Fig. 3b). Unlike in Fig. 2b, for temporal gradients we observe that the mean $\gamma$ value for a population of cells grows at rate $\alpha$, up to a critical value (in this case $\alpha \approx 1$ ), after which all cells die out (examples are presented in Figs. S2a-f, and the dependence of adaptation rate on $\alpha$ is plot in Fig. S3). This corresponds to the results of Lindsey et al, who found that cells were unable to adapt when the temporal rate of antibiotic increase was above a threshold value (Lindsey et al., 2013). This result also illustrates that in order to maintain a sub-maximal population density there is a narrow range of feasible rates of antibiotic increase with time. This was observed experimentally by Toprak et al. (Toprak et al., 2012), who used a temporal antibiotic gradient (which was approximately linear when plot on log-scale, corresponding here to $\gamma_{c} \propto t$ ) to regulate the density of an adapting bacterial population.

When encountering a large step rise in antibiotic concentration the majority of cells (with $\gamma \approx 0$ initially) rapidly die out, however, a few cells with large $\gamma$ overcome the step and return the population to its maximal density following a short proliferation delay (Fig. 3c). In such a circumstance it is possible for there to be a greater rate of adaptation (change in mean $\gamma$ value over time) for a short period than for antibiotic ramps (i.e. Fig. 2b), as predicted by Greulich et al. (Greulich et al., 2012). In Fig. 3d (left axis) we plot the probability of a given cell being able to 
replicate (i.e. having $\gamma>\gamma_{c}$ ) immediately following a step increase in $\gamma_{c}$, which is determined by the cumulative density function of the Laplace distribution from Eq. (2). The shape of this curve replicates typical killing curves observed for antibiotics (Liu et al., 2004) when plot as a linear function of antibiotic concentration (see Note S4). The point at which this curve drops to 0.001 $\left(\gamma_{c} / \phi \approx 4\right)$ approximates the MBC (minimum bactericidal concentration) of the antibiotic, which is generally defined as the concentration required to reduce $99.9 \%$ of bacterial density (Wang et al., 2016). The MIC (minimum inhibitory concentration) takes a similar value (Andersson and Hughes, 2014). The problem with using these metrics for measuring antibiotic efficacy (as discussed by Liu et al. (Liu et al., 2004)) is highlighted by the population survival curve (Fig. 3d right axis): Even though the vast majority of cells are killed above the $\mathrm{MBC}$, the survival of even a single cell with high resilience can re-establish the population. The point above which this does not occur (i.e. the point where the population survival probability drops to zero, here at $\gamma_{c} / \phi \approx 8$ ) is referred to as the MPC (mutant prevention concentration) (Wang et al., 2016). The distance between these curves represents the mutant selection window (MSW), a range of concentrations which is clinically important as it encourages selection for highly resistant mutants (Bell and MacLean, 2018).

In Fig. 3d we also plot changes anticipated in the Population Survival probability when either $D_{\gamma}$ or $\theta$ is varied while the step in antibiotic maintained at a constant multiple of $\phi$ (so that each cell survival probability curve is identical). Adjusting these parameters highlights the relative influence of two means via which a population can overcome a temporal step in $\gamma_{c}$ : The first is by having at least one cell with $\gamma>\gamma_{c}$ when antibiotic is introduced (often assumed to be the predominant route to resistance (Andersson and Hughes, 2014)), allowing the step to be overcome (this probability is plot in Fig. S4). The second is having $\gamma<\gamma_{c}$ immediately following antibiotic introduction, but evolving to a state with $\gamma>\gamma_{c}$ prior to cell death. If the former was the only mechanism then we would expect changes in $D_{\gamma}$ or $\theta$ (which determine $\phi$ ) to have no influence on the population survival probability for a step of fixed $\gamma_{c} / \phi$. This is not the case, demonstrating that some populations in which no cells are able to reproduce post-antibiotic addition can still recover. We can investigate this situation analytically (see Note S5), finding that the probability that a cell with coping ability $\gamma<\gamma_{c}$ will survive (i.e. it reaches $\gamma_{c}$ before dying) thereby sustaining the population is given by:

$$
p(\text { Population Survival })=\exp \left(-\frac{\nu \sqrt{2 D_{\gamma} \delta}}{\theta}\right)
$$

where $\nu$ is a dimensionless parameter measuring the distance between the cell's initial $\gamma$ and $\gamma_{c}$. The dependence of Eq. (6) on $\delta$ highlights the impact that the development of tolerant cell types (slowly dying in the presence of antibiotic (Brauner et al., 2016)) has upon the emergence of resistance (Cohen et al., 2013; Fridman et al., 2014; Levin-Reisman et al., 2017): Tolerant cell populations (those with a smaller value of $\delta$ which die off slowly (Brauner et al., 2017)) often develop as a pre-cursor to actual resistance (Levin-Reisman et al., 2017). Note S6 derives a similar expression to Eq. (6) for the case in which $\gamma_{c}$ depends linearly on time, demonstrating the antibiotic's inefficacy when its (initially large) concentration is reduced rapidly.

In Fig. 3e we plot the steady-state mean coping ability $(\mathbb{E}[\gamma])$ and standard deviation $(\sigma[\gamma])$ of a population of cells subjected to a constant $\gamma_{c}$ value. Substantial adaptation occurs well below the $\mathrm{MBC}\left(\gamma_{c} / \phi \approx 4\right.$ in Fig. $\left.3 \mathrm{~d}\right)$, and even below the $\mathrm{IC}_{50}\left(\gamma_{c}=0\right)$. There has been substantial debate regarding whether selection at low antibiotic concentrations is important (Jansen et al., 2013; 390 
Andersson and Hughes, 2014), and whether low-concentration treatments should be employed to minimise resistance evolution (Read et al., 2011; Kouyos et al., 2014). According to the classical MSW hypothesis selection is only relevant within the MSW region (Jansen et al., 2013), however, many studies have demonstrated that significant adaptation can occur in response to low antibiotic concentrations (Andersson and Hughes, 2014). Our simulations support the case for adaptation at low concentrations, though they predict that the degree of adaptation in this region will be limited (i.e. cells will not become resistant to very high antibiotic concentrations). This aligns with studies of evolutionary processes which have demonstrated that many traits (such as protein stability (Taverna and Goldstein, 2002)) will typically drift toward their minimum evolutionary requirement, as values much higher do not offer a selective benefit (Bloom et al., 2006). For the variability of $\gamma$ within a population Fig. 3e suggests the existence of two distinct regimes: When $\gamma_{c}<\mu$ we have $\sigma[\gamma] \approx \phi$ (following from Eq. (2)), though this decreases as $\gamma_{c} \rightarrow 0^{-}$due to cells with very low $\gamma$ values being unable to reproduce. When $\gamma_{c}$ grows beyond $\gamma_{c}=0$ there is a sharp rise in $\sigma[\gamma]$ to a new constant value of $\approx 1.7 \phi$, as the population's distribution of $\gamma$ values is now bounded below by $\gamma_{c}$. This behaviour is observed as a widening of the distribution in Fig. 1, highlighting our model's prediction that the distribution of $\gamma$ values in a population of cells growing in the presence of $\gamma_{c}>0$ will tend to have both an increased mean and standard deviation, making it more likely to adapt to a future rise in $\gamma_{c}$. That said, following any rise in antibiotic concentration there is a period during which $\sigma[\gamma]$ is reduced (Fig. S5) whilst the population re-establishes its genetic diversity (i.e. reaching a new steady state distribution of $\gamma$ ) .

In Fig. 3f we initialise a clonal population (in which all cells begin with $\gamma=0$ ) and allow cells to proliferate (and mutate) for a period $t_{p}$, before subjecting the population to a step rise in antibiotic concentration. We observe that it takes a substantial amount of time for sufficient intra-population variability in $\gamma$ to develop to enable the population to overcome the antibiotic step when it is encountered. This process is accelerated significantly when cells proliferate in the presence of a low antibiotic concentration $\left(\gamma_{c}=0\right)$, highlighting the clinical role that low concentrations can play in pre-adapting populations, thereby enabling them to overcome large antibiotic doses at later times. Related situations that include the temporary addition or removal (after a period of adaptation) probability remains at unity for a long period after antibiotic removal, highlighting one of the major clinical challenges posed by antibiotic resistance: Even in the absence of antibiotic a pathogen's return to sensitivity is slow (MacLean et al., 2010; Durão et al., 2018). In Fig. S6e we plot the probability that a population is able to overcome a large temporal step in antibiotic concentration as a function of the time it spends at an intermediate antibiotic concentration. An approximately linear rise is observed, with the probability of survival approaching unity as the time given for adaptation increases, again demonstrating that past environmental conditions can greatly affect a population's survivability following a rapid environmental change (as observed by Gonzalez and Bell (Gonzalez and Bell, 2013)).

\subsection{Multiple strategies for resistance}

For many antibiotics there is a range of potential mechanisms which can develop in combination to provide resistance (Palmer et al., 2018). For example, efflux pumps (which remove an antibiotic from the cell's interior), or mutation in the protein/s that directly interact with the antibiotic (thereby disrupting its binding) can both contribute to a cell's coping ability. Experimental studies have demonstrated that there are often a number of evolutionary trajectories which a cell can take to acquire resistance (Barbosa et al., 2017; Palmer et al., 2018), and that competition between 
different trajectories can impact the overall rate of adaptation (Ogbunugafor and Eppstein, 2016). In this section we employ our modelling framework to analyse a system in which there are multiple strategies competing to develop resistance.

We first simulate a population of cells growing in the presence of a temporal gradient of antibiotic concentration (e.g. as in Fig. 3a) for which there are two potential strategies for resistance, $\gamma_{1}$ and $\gamma_{2}$, and the total coping ability is given by $\bar{\gamma}=\gamma_{1}+\gamma_{2}$. Each strategy is assumed to have an equal ability to mutate over time $\left(D_{\gamma_{1}}=D_{\gamma_{2}}\right)$, but their relative drift rate is varied according to $\theta_{1}=\theta / \sqrt{1-\zeta}$ and $\theta_{2}=\theta / \sqrt{\zeta}$ such that $1 / \theta^{2}=1 / \theta_{1}^{2}+1 / \theta_{2}^{2}$. At steady-state (and in the absence of antibiotic, $\left.\gamma_{c}=-\infty\right) \operatorname{Var}[\bar{\gamma}]$ is thus independent of $\zeta$, allowing us to compare populations with equivalent initial distributions of coping ability. In Fig. 4a,b we find that when $\zeta=0.5$ both strategies adapt at half the rate of the temporal gradient (an example is illustrated in Fig. S7a). In the presence of very strong mean reversion for strategy $2(\zeta \rightarrow 0), \gamma_{2}$ does not develop at all (an example is illustrated in Fig. S7c), as would be expected from a resistance strategy with very high fitness cost when a better alternative is available. Interestingly, for intermediate $\zeta$ values $\left(\zeta \approx 10^{-2}\right)$ at which the mean adaptation rate of $\gamma_{2}$ approaches zero there is a reduced probability (Fig. 4a) that the population survives indefinitely: In this case population die out can occur (an example is illustrated in Fig. S7b) when $\gamma_{2}$ temporarily rises, reducing the pressure on $\gamma_{1}$ to adapt and thus leaving $\bar{\gamma}$ too far below $\gamma_{c}$ to keep up when $\gamma_{2}$ 's strong mean reversion drives it back toward $\mu_{2}=0$. This phenomenon is primarily observed for intermediate $\zeta$ values because when $\zeta \gg 10^{-2}$ selective pressure can overcome the mean reversion to allow $\gamma_{2}$ to permanently adapt, whereas for $\zeta \ll 10^{-2}$ it is very unlikely that $\gamma_{2}$ will deviate from zero at any point. In Fig. S7i,j an alternate situation is investigated, in which $\theta_{1}=\theta$ and $\theta_{2}=\eta \theta$; in this case when $\gamma_{2}$ has no mean reversion $(\eta \rightarrow 0) \gamma_{1}$ still develops, with only a slightly reduced rate.

We now set $\theta_{1}=\theta_{2}$, but vary the mutation rate of each strategy according to $D_{\gamma_{1}}=D_{\gamma} \sqrt{1-\xi}$ and $D_{\gamma_{2}}=D_{\gamma} \sqrt{\xi}$, such that $D_{\gamma}^{2}=D_{\gamma_{1}}^{2}+D_{\gamma_{2}}^{2}$. We observe (Fig. 4c) that as $\xi$ grows our population goes from developing just one strategy to favouring a combination of both. In Fig. 4d we examine the influence of $\zeta$ or $\xi$ on the probability of $\gamma_{1}$ developing when the population is confronted with a temporal step in antibiotic concentration (i.e. as in Fig. 3c). We observe that outside of a narrow switching region there is a strong bias in favour of whichever strategy has a greater mutation rate (larger $D_{\gamma_{i}}$ ) or smaller rate of mean reversion / fitness cost (smaller $\theta_{i}$ ). In each case the winning resistance strategy develops largely to the exclusion of the other, such that $\max \left(\gamma_{1}, \gamma_{2}\right) \approx \bar{\gamma}$ and $\min \left(\gamma_{1}, \gamma_{2}\right) \approx 0$ (demonstrated in Fig. S7d,e). A similar result is observed when simulations are initialilsed with one strategy already dominating $\left(\mathbb{E}\left[\gamma_{1}\right]>\gamma_{c}>\mathbb{E}\left[\gamma_{2}\right]\right.$ at $t=0$, Fig. S7f,g): In this case if strategy $\gamma_{2}$ is "better" than $\gamma_{1}$ (large $\xi$ or $\zeta$ ) then it will (eventually) overcome $\gamma_{1}$ 's early lead and take over the population. This result highlights an interesting difference between temporal steps and ramps; for a step the better strategy will eventually dominate in the population (regardless of initial conditions), whereas if the population is driven to continually increase $\gamma$ (i.e. a temporal ramp) there is a broad parameter regime in which both strategies develop.

In Fig. 4e,f simulations (which are otherwise similar to Fig. 4b,c) are performed for a population of cells that encounters a spatial (rather than temporal) ramp of antibiotic concentration. In this case there is no possibility of the population dying out (as regions of low antibiotic concentration are always accessible), but there is a minimum in the adaptation rate of $\bar{\gamma}$ at $\zeta \approx 10^{-2}$ for similar reasons to Fig. 4a. We also observe that the inclusion of a second strategy (when $\operatorname{Var}[\bar{\gamma}]$ is kept constant) only results in a modest increase in the adaptation rate of $\bar{\gamma}$. Our results thus imply 
that when resistance is determined by multiple orthogonal strategies adaptation can occur more

\section{Discussion}

In this paper we have developed a theoretical model that describes the evolution of resistance in response to spatially- or temporally-varying antibiotic concentrations. Though both kinds of inhomogeneities can foster the development of high levels of resistance, they are not necessarily the time of dosing in order for the population to survive (this is often assumed to be the predominant route to resistance (Andersson and Hughes, 2014)), though there is the rare possibility of a "tolerance" path to population resistance, as described by Eq. (6). For spatial steps in antibiotic this path to resistance is less important. Instead, cells can undergo continual variation in $\gamma$ near a discontinuity in their environment (a point at which $\gamma_{c}$ increases), and once a mutant with $\gamma>\gamma_{c}$ appears they quickly migrate into the now accessible resource-rich region. This corresponds to experimental observations that the evolutionary process is not driven by its fittest constituents, but rather those that are both sufficiently fit and sufficiently close to an environmental inhomogeneity (Baym et al., 2016a). The overcoming of spatial inhomogeneities is aided by the fact that there accessible regions of smaller $\gamma_{c}$ in which cells can proliferate, which fundamentally changes the dynamics of competition between multiple resistance strategies in response to each kind of inhomogeneity. For example, we found that the presence of a second adaptive strategy for resistance can lead to a population's extinction in the presence of an otherwise manageable temporal antibiotic gradient (but not for a similar spatial gradient). Furthermore, when cells are presented with temporal steps in antibiotic concentration typically one strategy will be developed to the exclusion of others, whereas a combination of resistance strategies is likely to evolve in response to spatial steps.

The rate at which resistance increases in time $(\mathrm{d} \gamma / \mathrm{d} t)$ also differs significantly for the two classes magnitude $(\mathrm{d} \gamma / \mathrm{d} t \approx \alpha)$ up to a limiting $\alpha$ where population extinction occurs. Meanwhile, for spatial gradients adaptation rate is determined by a trade-off between spatial diffusion (limiting for small $\alpha$ ) and the population size near to an accessible (via small $\gamma$ increase) region of larger $\gamma_{c}$ (limiting for large $\alpha$ ). These results highlight the importance of avoiding intermediate antibiotic doses which, if they produce intermediate spatial/temporal concentration gradients, maximise the rate at which resistance emerges.

Persister and tolerant cell states have been recognised as playing a prominent part in the development of resistance (Brauner et al., 2016). Our model (see Eq. (6)) supports the experimental likelihood of resistance developing (Cohen et al., 2013; Fridman et al., 2014; Levin-Reisman et al., 2017). However, since the death rate $(\delta)$ is assumed to be homogenous within each population, we do not account for the wide range of persister behaviours that have been experimentally observed 
(Brauner et al., 2016): In such cases a small fraction of a clonal cell population may remain dormant in the presence of an antibiotic (via a range of mechanisms (Harms et al., 2016)), allowing them to re-establish the entire population when conditions are more favourable. To capture this behaviour our model would likely need to introduce additional parameters to provide a more complex description of cell states, or separate cells into sub-populations with differing parameter values.

At the level of individual cells, our modelling approach assumes that antibiotic resistance develops in a continuous manner (governed by Eq. (2)) as is frequently observed experimentally (Toprak et al., 2012; Chevereau et al., 2015; Barrick and Lenski, 2013; Palmer et al., 2018). However, in some cases resistance can develop in jumps due to distinct high-impact mutations (Toprak et al., 2012; Chevereau et al., 2015; Barrick and Lenski, 2013) (or by transfer of genes via plasmids). This possibility is investigated in Note S7, where qualitatively similar results to the continuous case are found when $\gamma$ is constrained to integer multiples of a discretisation parameter $\omega$. Though both treatments of $\gamma$ allow populations as a whole to make large non-continuous jumps in resistance (e.g. as in Fig. 3c), additional parameters would be required to account for the complex mutational landscapes found in practice for some antibiotics: This might (for example) include defining $D_{\gamma}$ as an explicit function of $\gamma$ to account for features such as fitness valleys in adaptive processes (Greulich et al., 2012) though any such function would be highly antibiotic- and resistance strategy-dependent (Ogbunugafor and Eppstein, 2016; Bollenbach, 2015). Likewise, in some cases an upper bound on the effectiveness of certain resistance strategies may be present (Toprak et al., 2012; Lukačišinová and Bollenbach, 2017), and (for adaptive evolution in general) certain paths may lead to sub-optimal outcomes (Dickinson et al., 2013). Models similar to ours may be useful in describing the trapping of evolution in sub-optimal fitness states (de Vos et al., 2013), which may be realised experimentally using chemical/environmental factors to steer adaptive processes down sub-optimal paths (Furusawa et al., 2018; Gifford et al., 2018).

In recent years it has become apparent that the development of bacterial resistance to antibiotics can be significantly accelerated by spatiotemporal variation in antibiotic concentration. We have developed a mathematical description of these processes, and applied it to analyse a diverse range of experimental results. Our model provides a framework for informing and analysing future studies of the evolution of antibiotic resistance, and may be applicable to many similar adaptive processes in which the rate of fitness cost-driven drift towards a wild type state (here described by $\theta$ ) can 560 be assumed to be independent of the trait's value $(\gamma)$.

\section{Competing Interests}

The authors declare no competing interests.

\section{Acknowledgements}

H. Steel is supported by the General Sir John Monash Foundation. A. Papachristodoulou is 565 supported by EPSRC project EP/M002454/1.

\section{References}

Amitai, G., Gupta, R. D., and Tawfik, D. S., 2007. Latent evolutionary potentials under the neutral mutational drift of an enzyme. HFSP Journal, 1:67-78. 
Andersson, D. I., 2006. The biological cost of mutational antibiotic resistance: any practical conclusions? Current Opinion in Microbiology, 9:461-465.

Andersson, D. I. and Hughes, D., 2014. Microbiological effects of sublethal levels of antibiotics. Nature Reviews Microbiology, 12:465-478.

Barbosa, C., Trebosc, V., Kemmer, C., Rosenstiel, P., Beardmore, R., Schulenburg, H., and Jansen, G., 2017. Alternative Evolutionary Paths to Bacterial Antibiotic Resistance Cause Distinct Collateral Effects. Molecular biology and evolution, 34:2229-2244.

Barrick, J. E. and Lenski, R. E., 2013. Genome dynamics during experimental evolution. Nature Reviews Genetics, 14:827-839.

Baym, M., Lieberman, T., Kelsic, E., Chait, R., Gross, R., Yelin, I., and Kishony, R., $2016 a$. Spatiotemporal microbial evolution on antibiotic landscapes. Science, 353:1147-1152.

Baym, M., Stone, L. K., and Kishony, R., 2016b. Multidrug evolutionary strategies to reverse antibiotic resistance. Science, 351.

Bean, D. C., Livermore, D. M., Papa, I., and Hall, L. M., 2005. Resistance among Escherichia coli to sulphonamides and other antimicrobials now little used in man. Journal of Antimicrobial Chemotherapy, 56:962-964.

Beaulieu, J. M., Jhwueng, D. C., Boettiger, C., and O'Meara, B. C., 2012. Modeling stabilizing selection: Expanding the Ornstein-Uhlenbeck model of adaptive evolution. Evolution, 66:23692383 .

Beerenwinkel, N., Antal, T., Dingli, D., Traulsen, A., Kinzler, K. W., Velculescu, V. E., Vogelstein, B., and Nowak, M. A., 2007. Genetic Progression and the Waiting Time to Cancer. PLoS Computational Biology, 3.

Behrman, K. D. and Kirkpatrick, M., 2011. Species range expansion by beneficial mutations. Journal of Evolutionary Biology, 24:665-675.

Bell, G. and MacLean, C., 2018. The Search for Evolution-Proof'Antibiotics. Trends in Microbiology, 26:471-483.

Blomberg, S., 2012. Beyond Brownian motion and the Ornstein-Uhlenbeck process: Stochastic diffusion models for the evolution of quantitative characters. BioRxiv.

Bloom, J. D. and Arnold, F. H., 2009. In the light of directed evolution: Pathways of adaptive protein evolution. Proceedings of the National Academy of Sciences, 106:9995-10000.

Bloom, J. D., Labthavikul, S. T., Otey, C. R., and Arnold, F. H., 2006. Protein stability promotes evolvability. Proceedings of the National Academy of Sciences, 103:5869-5874.

Bloom, J. D., Romero, P. A., Lu, Z., and Arnold, F. H., 2007. Neutral genetic drift can alter promiscuous protein functions, potentially aiding functional evolution. Biology Direct, 2:7-10.

Bollenbach, T., 2015. Antimicrobial interactions: Mechanisms and implications for drug discovery and resistance evolution. Current Opinion in Microbiology, 27:1-9. 
Brauner, A., Fridman, O., Gefen, O., and Balaban, N. Q., 2016. Distinguishing between resistance, tolerance and persistence to antibiotic treatment. Nature Reviews Microbiology, 14:320-330.

Brauner, A., Shoresh, N., Fridman, O., and Balaban, N. Q., 2017. An Experimental Framework for Quantifying Bacterial Tolerance. Biophysical Journal, 112:2664-2671.

Bridle, J. R. and Vines, T. H., 2007. Limits to evolution at range margins: when and why does 610 adaptation fail? Trends in Ecology and Evolution, 22:140-147.

Chevereau, G., Dravecká, M., Batur, T., Guvenek, A., Ayhan, D. H., Toprak, E., and Bollenbach, T., 2015. Quantifying the Determinants of Evolutionary Dynamics Leading to Drug Resistance. PLoS Biology, 13:1-18.

Cohen, N. R., Lobritz, M. A., and Collins, J. J., 2013. Microbial persistence and the road to drug 615 resistance. Cell Host and Microbe, 13:632-642.

De Gelder, L., Ponciano, J. M., Abdo, Z., Joyce, P., Forney, L. J., and Top, E. M., 2004. Combining mathematical models and statistical methods to understand and predict the dynamics of antibiotic-sensitive mutants in a population of resistant bacteria during experimental evolution. Genetics, 168:1131-1144.

De Jong, M. G. and Wood, K. B., 2018. Tuning Spatial Profiles of Selection Pressure to Modulate the Evolution of Drug Resistance. Physical Review Letters, 120:238102.

de Vos, M. G. J., Poelwijk, F. J., and Tans, S. J., 2013. Optimality in evolution: New insights from synthetic biology. Current Opinion in Biotechnology, 24:797-802.

Deris, J. B., Kim, M., Zhang, Z., Okano, H., Hermsen, R., Groisman, A., and Hwa, T., 2013. The ${ }^{625}$ innate growth bistability and fitness landscapes of antibiotic-resistant bacteria. Science, 342.

Dickinson, B. C., Leconte, A. M., Allen, B., Esvelt, K. M., and Liu, D. R., 2013. Experimental interrogation of the path dependence and stochasticity of protein evolution using phage-assisted continuous evolution. Proceedings of the National Academy of Sciences, 110:9007-9012.

Didelot, X., Walker, A. S., Peto, T. E., Crook, D. W., and Wilson, D. J., 2016. Within-host ${ }^{630}$ evolution of bacterial pathogens. Nature Reviews Microbiology, 14:150-162.

Droz, M. and Pkalski, A., 2006. Population dynamics in heterogeneous conditions. Physica A: Statistical Mechanics and its Applications, 362:504-512.

Durão, P., Balbontín, R., and Gordo, I., 2018. Evolutionary Mechanisms Shaping the Maintenance of Antibiotic Resistance. Trends in Microbiology, 26:677-691.

Erickson, K. E., Otoupal, P. B., and Chatterjee, A., 2016. Gene Expression Variability Underlies Adaptive Resistance in Phenotypically Heterogeneous Bacterial Populations. ACS Infectious Diseases, 1:555-567.

Feng, Y., Jonker, M. J., Moustakas, I., Brul, S., and Ter Kuile, B. H., 2016. Dynamics of mutations during development of resistance by Pseudomonas aeruginosa against five antibiotics. ${ }_{640}$ Antimicrobial Agents and Chemotherapy, 60:4229-4236. 
Franke, J., Klozer, A., de Visser, J. A. G., and Krug, J., 2011. Evolutionary Accessibility of Mutational Pathways. PLoS Computational Biology, 7.

Fridman, O., Goldberg, A., Ronin, I., Shoresh, N., and Balaban, N. Q., 2014. Optimization of lag time underlies antibiotic tolerance in evolved bacterial populations. Nature, 513:418-421.

Furusawa, C., Horinouchi, T., and Maeda, T., 2018. Toward prediction and control of antibioticresistance evolution. Current Opinion in Biotechnology, 54:45-49.

Gerrish, P. J. and García-Lerma, J. G., 2003. Mutation rate and the efficacy of antimicrobial drug treatment. The Lancet Infectious Diseases, 3:28-32.

Gifford, D. R., Furió, V., Papkou, A., Vogwill, T., Oliver, A., and MacLean, R. C., 2018. Identifying and exploiting genes that potentiate the evolution of antibiotic resistance. Nature Ecology and Evolution, 2:1033-1039.

Gillespie, J., 1984. Molecular Evolution Over the Mutational Landscape. Evolution, 38:1116-1129.

Gonzalez, A. and Bell, G., 2013. Evolutionary rescue and adaptation to abrupt environmental change depends upon the history of stress. Philosophical Transactions of the Royal Society B: Biological Sciences, 368.

Gould, I. M. and MacKenzie, F. M., 2002. Antibiotic exposure as a risk factor for emergence of resistance: the influence of concentration. J.Appl.Microbiol., 92:78S-84S.

Gralka, M., Fusco, D., Martis, S., and Hallatschek, O., 2017. Convection shapes the trade-off between antibiotic efficacy and the selection for resistance in spatial gradients. Physical Biology, 14.

Greulich, P., Waclaw, B., and Allen, R. J., 2012. Mutational pathway determines whether drug gradients accelerate evolution of drug-resistant cells. Physical Review Letters, 109:1-5.

Hansen, T. F., 1997. Stabilizing selection and the comparative analysis of adaptation. Evolution, $51: 1341-1351$.

Harms, A., Maisonneuve, E., and Gerdes, K., 2016. Mechanisms of bacterial persistence during stress and antibiotic exposure. Science, 354.

Hermsen, R., 2016. The adaptation rate of a quantitative trait in an environmental gradient. Physical Biology, 13.

Hermsen, R., Deris, J. B., and Hwa, T., 2012. On the rapidity of antibiotic resistance evolution facilitated by a concentration gradient. Proceedings of the National Academy of Sciences, 109: 10775-10780.

Hindré, T., Knibbe, C., Beslon, G., and Schneider, D., 2012. New insights into bacterial adaptation through in vivo and in silico experimental evolution. Nature Reviews Microbiology, 10:352-365.

Hol, F. J., Hubert, B., Dekker, C., and Keymer, J. E., 2016. Density-dependent adaptive resistance allows swimming bacteria to colonize an antibiotic gradient. ISME Journal, 10:30-38. 
Jacobs, M., Grégoire, N., Couet, W., and Bulitta, J. B., 2016. Distinguishing Antimicrobial Models with Different Resistance Mechanisms via Population Pharmacodynamic Modeling. PLoS Computational Biology, 12:1-19.

Jansen, G., Barbosa, C., and Schulenburg, H., 2013. Experimental evolution as an efficient tool 680 to dissect adaptive paths to antibiotic resistance. Drug Resistance Updates, 16:96-107.

Kepler, T. B. and Perelson, A. S., 1998. Drug concentration heterogeneity facilitates the evolution of drug resistance. Proceedings of the National Academy of Sciences, 95:11514-11519.

Kohanski, M., Dwyer, D., and Collins, J. J., 2010. How antibiotics kill bacteria: from targets to networks. Nature Reviews Microbiology, 08:423-435.

Kouyos, R. D., Metcalf, C. J. E., Birger, R., Klein, E. Y., zur Wiesch, P. A., Ankomah, P., Arinaminpathy, N., Bogich, T. L., Bonhoeffer, S., Brower, C., Chi-Johnston, G., Cohen, T., Day, T., Greenhouse, B., Huijben, S., Metlay, J., Mideo, N., Pollitt, L. C., Read, A. F., Smith, D. L., Standley, C., Wale, N., and Grenfell, B., 2014. The path of least resistance: Aggressive or moderate treatment? Proceedings of the Royal Society B: Biological Sciences, 281.

Lande, R., 1976. Natural Selection and Random Genetic Drift in Phenotypic Evolution. Evolution, 30:314-334.

Levin-Reisman, I., Ronin, I., Gefen, O., Braniss, I., Shoresh, N., and Balaban, N. Q., 2017. Antibiotic tolerance facilitates the evolution of resistance. Science, 355:826-830.

Lindsey, H. A., Gallie, J., Taylor, S., and Kerr, B., 2013. Evolutionary rescue from extinction is 695 contingent on a lower rate of environmental change. Nature, 494:463-467.

Ling, L. L., Schneider, T., Peoples, A. J., Spoering, A. L., Engels, I., Conlon, B. P., Mueller, A., Schäberle, T. F., Hughes, D. E., Epstein, S., Jones, M., Lazarides, L., Steadman, V. A., Cohen, D. R., Felix, C. R., Fetterman, K. A., Millett, W. P., Nitti, A. G., Zullo, A. M., Chen, C., and Lewis, K., 2015. A new antibiotic kills pathogens without detectable resistance. Nature, 517: 700 455-459.

Liu, Y. Q., Zhang, Y. Z., and Gao, P. J., 2004. Novel Concentration-Killing Curve Method for Estimation of Bactericidal Potency of Antibiotics in an In Vitro Dynamic Model. Antimicrobial Agents and Chemotherapy, 48:3884-3891.

Lukačišinová, M. and Bollenbach, T., 2017. Toward a quantitative understanding of antibiotic 705 resistance evolution. Current Opinion in Biotechnology, 46:90-97.

MacLean, R. C., Hall, A. R., Perron, G. G., and Buckling, A., 2010. The population genetics of antibiotic resistance: Integrating molecular mechanisms and treatment contexts. Nature Reviews Genetics, 11:405-414.

McDonald, M. J., Cooper, T. F., Beaumont, H. J. E., and Rainey, P. B., 2011. The distribution 710 of fitness effects of new beneficial mutations in Pseudomonas fluorescens. Biol. Lett., 7:98-100.

Moura De Sousa, J., Sousa, A., Bourgard, C., and Gordo, I., 2015. Potential for adaptation overrides cost of resistance. Future Microbiology, 10:1415-1431. 
Mozhayskiy, V. and Tagkopoulos, I., 2013. Microbial evolution in vivo and in silico: Methods and 715 applications. Integrative Biology, 5:262-277.

Ogbunugafor, C. B. and Eppstein, M. J., 2016. Competition along trajectories governs adaptation rates towards antimicrobial resistance. Nature Ecology \& Evolution, 1.

Opatowski, L., Guillemot, D., Boëlle, P. Y., and Temime, L., 2011. Contribution of mathematical modeling to the fight against bacterial antibiotic resistance. Current Opinion in Infectious Diseases, 24:279-287.

Oz, T., Guvenek, A., Yildiz, S., Karaboga, E., Tamer, Y. T., Mumcuyan, N., Ozan, V. B., Senturk, G. H., Cokol, M., Yeh, P., and Toprak, E., 2014. Strength of selection pressure is an important parameter contributing to the complexity of antibiotic resistance evolution. Molecular Biology and Evolution, 31:2387-2401.

Pál, C., Papp, B., and Lázár, V., 2015. Collateral sensitivity of antibiotic-resistant microbes. Trends in Microbiology, 23:401-407.

Palmer, A. C., Toprak, E., Baym, M., Kim, S., Veres, A., Bershtein, S., and Kishony, R., 2015. Delayed commitment to evolutionary fate in antibiotic resistance fitness landscapes. Nature Communications, 6:1-8.

Palmer, A. C., Chait, R., and Kishony, R., 2018. Non-optimal gene expression creates latent potential for antibiotic resistance. Molecular Biology and Evolution.

Pennell, M. W., FitzJohn, R. G., Cornwell, W. K., and Harmon, L. J., 2015. Model Adequacy and the Macroevolution of Angiosperm Functional Traits. The American Naturalist, 186:E33-E50.

Posada, D. and Crandall, K., 2001. Selecting the Best-Fit Model of Nucleotide Substitution. Systematic Biology, 50:580-601.

Read, A. F., Day, T., and Huijben, S., 2011. The evolution of drug resistance and the curious orthodoxy of aggressive chemotherapy. Proceedings of the National Academy of Sciences of the United States of America, 108:10871-10877.

Rokyta, D. R., Joyce, P., Caudle, S. B., and Wichman, H. A., 2005. An empirical test of the mutational landscape model of adaptation using a single-stranded DNA virus. Nature Genetics, $37: 441-444$.

Schenk, M. F., Szendro, I. G., Krug, J., and de Visser, J. A. G., 2012. Quantifying the adaptive potential of an antibiotic resistance enzyme. PLoS Genetics, 8.

Schrag, S. J., Perrot, V., and Levin, B. R., 1997. Adaptation to the fitness costs of antibiotic resistance in Escherichia coli. Proc Biol Sci, 264:1287-1291.

Simonsen, M., Leth, J., Schioler, H., and Cornean, H., 2013. A Simple Stochastic Differential Equation with Discontinuous Drift. Electronic Proceedings in Theoretical Computer Science, 124:109-123.

Soothill, J. S., Ward, R., and Girling, A. J., 1992. The IC50: An exactly defined measure of antibiotic sensitivity. Journal of Antimicrobial Chemotherapy, 29:137-139. 
Spicknall, I. H., Foxman, B., Marrs, C. F., and Eisenberg, J. N., 2013. A modeling framework for the evolution and spread of antibiotic resistance: Literature review and model categorization. American Journal of Epidemiology, 178:508-520.

Taverna, D. M. and Goldstein, R. A., 2002. Why are proteins marginally stable? Proteins: Structure, Function and Genetics, 46:105-109.

Toprak, E., Veres, A., Michel, J. B., Chait, R., Hartl, D. L., and Kishony, R., 2012. Evolutionary paths to antibiotic resistance under dynamically sustained drug selection. Nature Genetics, 44: 101-105.

Uyeda, J. C. and Harmon, L. J., 2014. A novel Bayesian method for inferring and interpreting the dynamics of adaptive landscapes from phylogenetic comparative data. Systematic Biology, 63: 760 902-918.

Wang, J., Hao, H., Huang, L., Liu, Z., Chen, D., and Yuan, Z., 2016. Pharmacokinetic and pharmacodynamic integration and modeling of enrofloxacin in swine for Escherichia coli. Frontiers in Microbiology, 7.

Weinreich, D. M., Delaney, N. F., Depristo, M. a., and Hartl, D. L., 2006. Darwinian Evolution 765 Can Follow Only Very Few Mutational Paths to Fitter Proteins. Science, 312:2004-2007.

Zampieri, M., Enke, T., Chubukov, V., Ricci, V., Piddock, L., and Sauer, U., 2017. Metabolic constraints on the evolution of antibiotic resistance. Molecular Systems Biology, 13:917.

Zhang, Q., Lambert, G., Liao, D., Kim, H., Robin, K., Tung, C.-k., Pourmand, N., and Austin, R. H., 2011. Acceleration of Emergence of Bacterial Antibiotic Resistance in Connected Mi- 770 croenvironments. Science, pages 1764-1767.

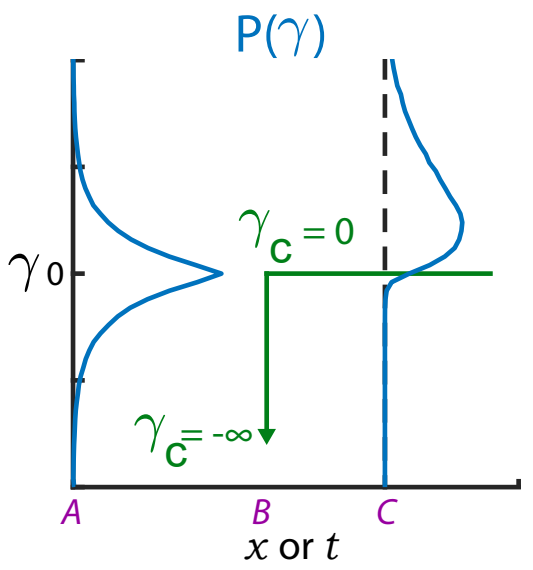

Figure 1: Probability distributions of coping ability. At point $A$ no antibiotic is present $\left(\gamma_{c}=-\infty\right)$ and the stationary distribution of coping ability $(P(\gamma))$ within a population of cells is Laplacian as predicted by Eq. (2). At point $B$ antibiotic is added such that $\gamma_{c}=0$, immediately following which cells with $\gamma<0$ are unable to replicate. At a later point in time/distance $C$ a new stationary distribution has been established, no longer Laplacian since selection for resistance is occurring, and hence selective reproduction interferes with the stationary distribution predicted by Eq. (2). Note that at point $C$ some (small number of) cells have $\gamma<\gamma_{c}$ due to ongoing mutation, and the population's mean $\gamma$ is substantially greater than $\gamma_{c}$. 

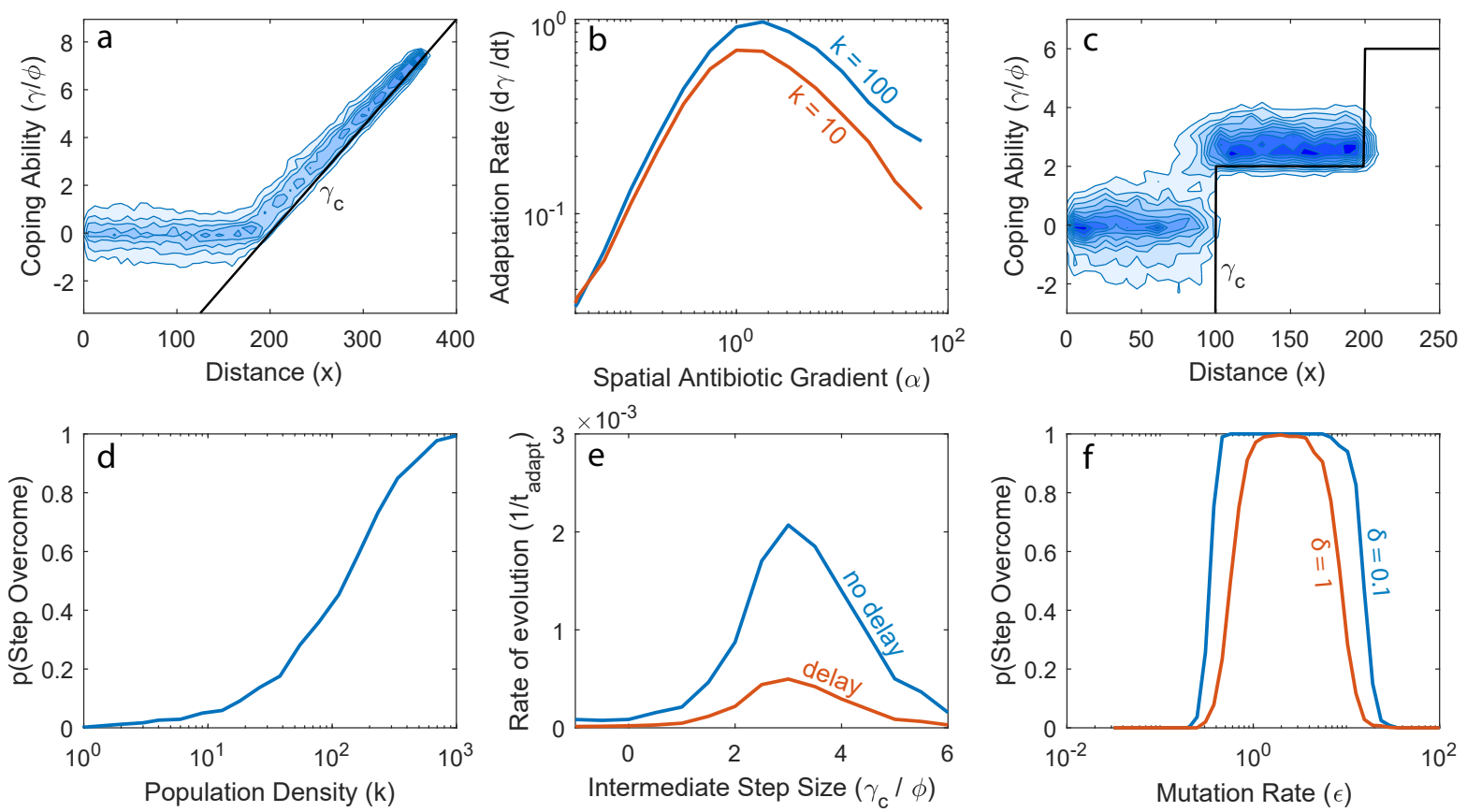

Figure 2: Spatial antibiotic variation encourages development of resistance. a) Probability density level curves of a population of cells in terms of coping ability $\gamma$ and position $x$ as they evolve in the presence of a spatial gradient in antibiotic $\gamma_{c}=x-200$. b) The adaptation rate as a function of spatial antibiotic gradient, $\gamma_{c}=\alpha x$, is maximised when $\alpha \approx 1$. Simulation details are presented in Note S8, and examples of adaptation with varying $\alpha$ are presented in Fig. S1a-c. c) Probability density level curves of a population evolving in response to two spatial steps in antibiotic concentration. For $x<100 \gamma_{c}=-\infty$, for $100<x<200 \gamma_{c}=2 \phi$, and for $x>200$ $\gamma_{c}=6 \phi$. The population is able to overcome the first step, but not the second. d) Probability of a fixed step in antibiotic concentration being overcome as a function of spatial population density $k$ (Note S9). A similar plot as a function of $\gamma_{c}$ is provided in Fig. S1d e) The rate of evolution in a two-step system (similar to c) as a function of the intermediate step size (Note S11). Also plot is the effect of adding a delay to account for extended spatial separation between the steps. f) The probability of a single step in antibiotic concentration being overcome as a function of an organism-wide mutation rate disturbance $\epsilon$, with different values of $\delta$ (Note S12). There exists an optimal region of intermediate $\epsilon$ where this probability is maximised. 

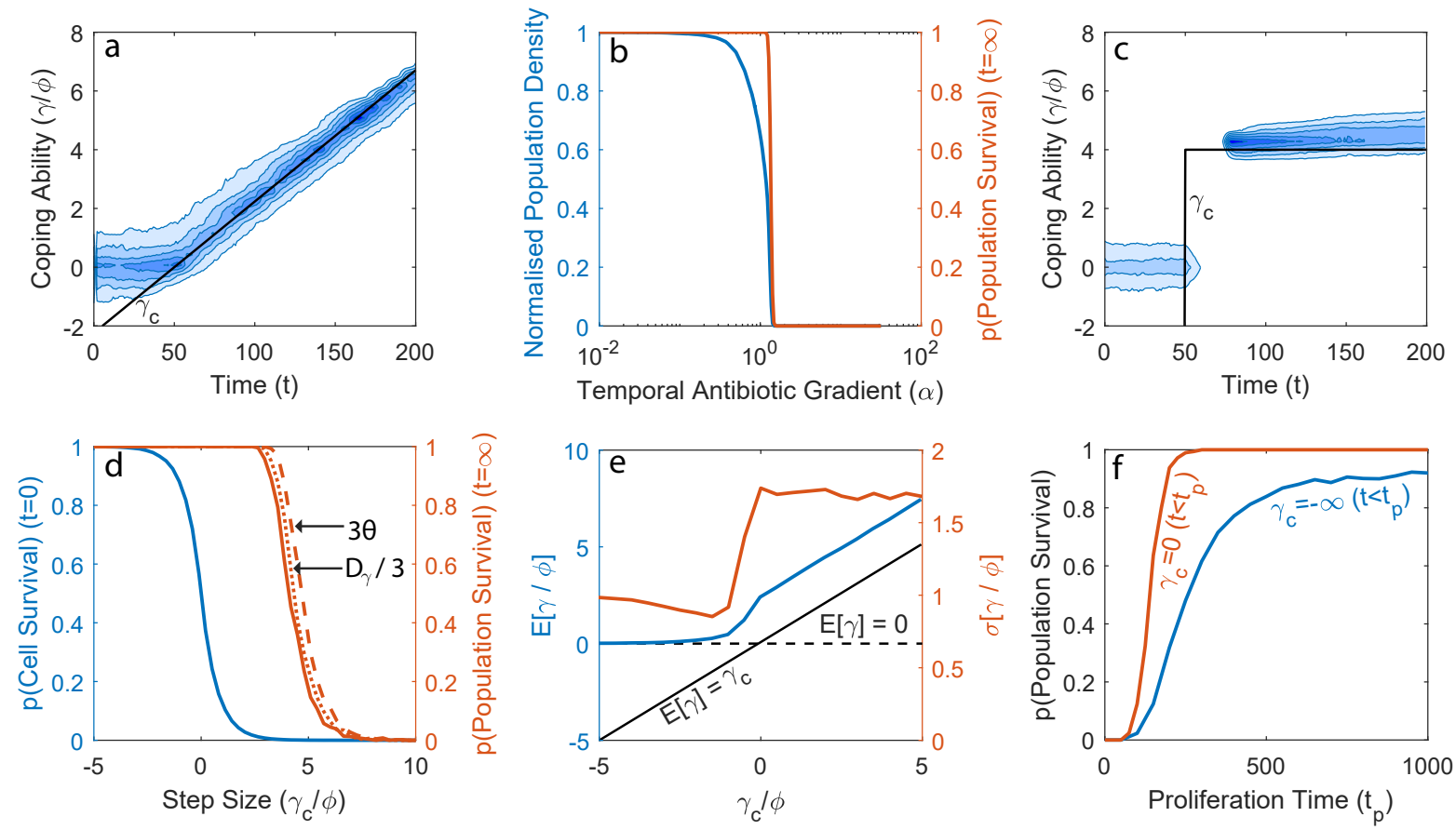

Figure 3: Temporal antibiotic variation encourages development of resistance. a) Probability density level curves as a function of time for cells evolving in the presence of a temporal gradient in antibiotic concentration $\gamma_{c}=t-50$. Here we are plotting a population of cells at a single location at many points in time (rather than a population of cells at many locations at a single point in time as in Fig. 2a). b) The normalised population density (left axis) and the probability of a population of cells being able to survive indefinitely (right axis) as a function of the temporal gradient in antibiotic concentration (with $\gamma_{c}=\alpha t$ ). Simulation details are presented in Note S13, and examples of cell populations growing with various $\alpha$ are in Fig. S2a-f. c) Probability density level curves as a function of time for cells that encounter a step rise in antibiotic. For $t<50 \gamma_{c}=-\infty$, and for $t>50 \gamma_{c}=4 \phi$. The introduction of antibiotic kills almost all cells at $t=50$, but survivors have re-established a large population by $t \approx 70 . \mathbf{d}$ ) The probability of a single cell surviving (left axis) and the population surviving (right axis) as a function of the size of an antibiotic step rise $\gamma_{c}$ (Note S14). We vary the parameters $\theta$ and $D_{\gamma}$ between trials, observing that the population survival probability changes, though the probability of a given cell surviving remains unchanged (because $\phi$ varies when $\theta$ or $D_{\gamma}$ change). e) The mean $(\mathbb{E}[\gamma / \phi])$ and standard deviation $(\sigma[\gamma / \phi])$ of $\gamma$ for a population of cells allowed to evolve for a long period in the presence of a fixed $\gamma_{c}$ (Note S15). f) The probability of a clonal population (initially all cells have $\gamma=0$ ) surviving a step rise in antibiotic concentration following a period of proliferation $\left(t_{p}\right)$ during which either $\gamma_{c}=-\infty$ or $\gamma_{c}=0$ (Note S17). 

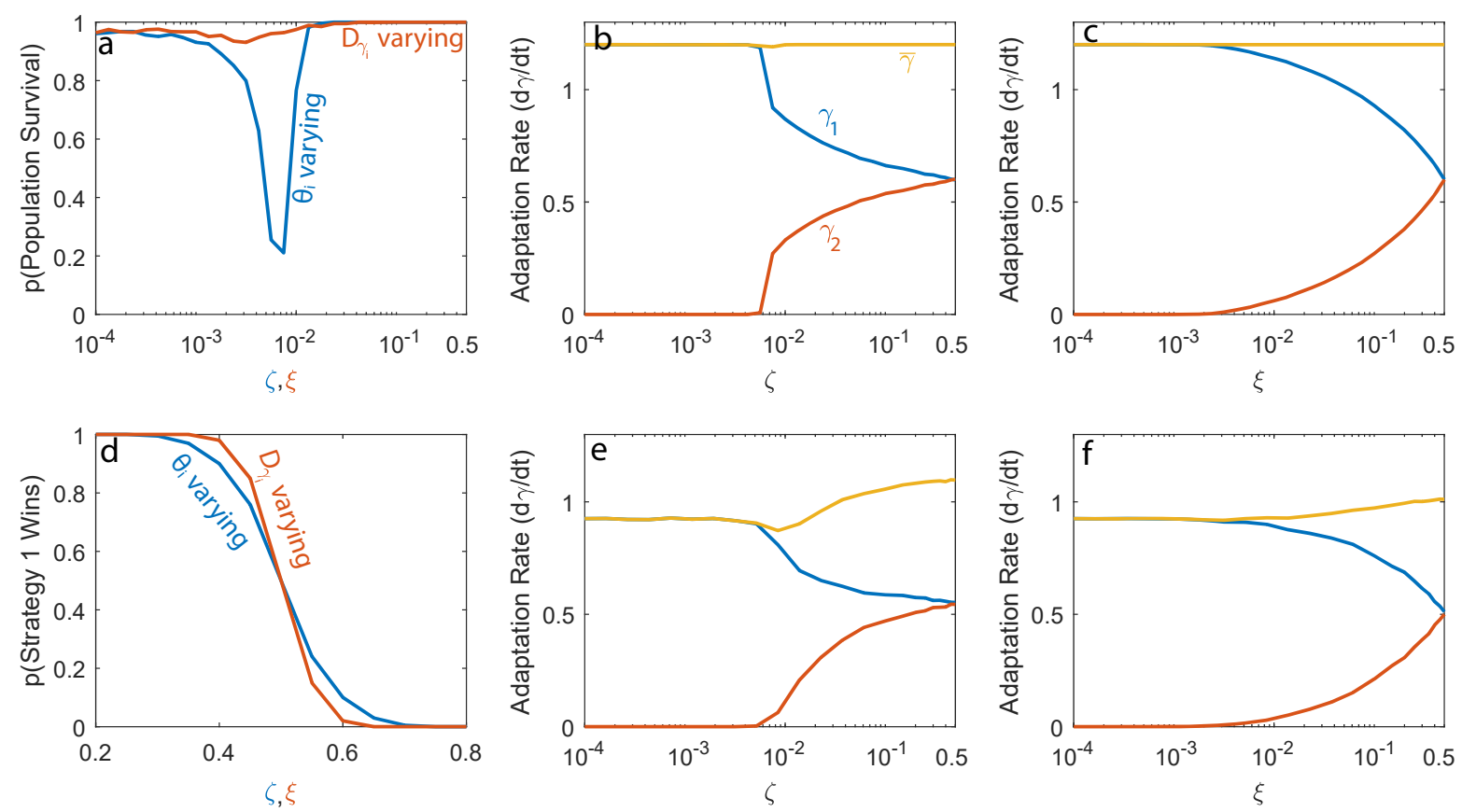

Figure 4: Competition between multiple paths to antibiotic resistance. We consider two strategies $\gamma_{1}$ and $\gamma_{2}$ which combine as described by Eq. (5), with either $\theta_{1}=\theta / \sqrt{1-\zeta}$ and $\theta_{2}=\theta / \sqrt{\zeta}$, or $D_{\gamma_{1}}=D_{\gamma} \sqrt{1-\xi}$ and $D_{\gamma_{2}}=D_{\gamma} \sqrt{\xi}$. a) The probability of the population's survival when subjected to a temporal ramp $(\alpha=1.2)$ in antibiotic concentration as a function of the second strategy's rate of mean reversion. For intermediate values of $\zeta$ there is a significant probability that the second strategy causes population die out (Note S21). Examples for various $\xi$ are presented in Fig. S7a-c. b,c) The adaptation rate (defined as in Fig. 2b) of each strategy (labelling is consistent across subplots b,c,e,f) as a function of $\zeta$ or $\xi$ (Note S21). The combined rate of change in $\bar{\gamma}$ is constant and equal to the temporal rate of change of antibiotic $(\alpha=1)$. d) The probability that Strategy 1 wins (defined as having a larger mean $\gamma_{i}$ value across the population) when the population is subjected to a temporal step in antibiotic concentration (Note S22), as a function of $\zeta$ (top axis) and $\xi$ (bottom axis). e,f) Similar to b,c but for a population of cells growing on a spatial antibiotic gradient with $\alpha=1$ (Note S25). 
The effect of spatiotemporal antibiotic inhomogeneities on the evolution of resistance

\author{
Supplementary Material
}

Harrison Steel and Antonis Papachristodoulou 


\section{Contents}

Figure S1

Supplementary Figures $\quad 2$

Figure S2 . . . . . . . . . . . . . . . . . . . . . . . . . . . . . . . . . 3

Figure S3 . . . . . . . . . . . . . . . . . . . . . . . . . . . . . . . . . 4

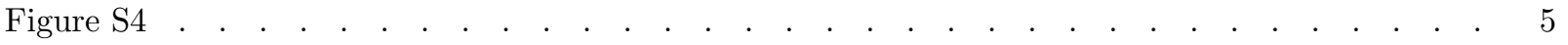

Figure S5 . . . . . . . . . . . . . . . . . . . . . . . . . . . . . . . . . . . . . . . .

Figure S6 . . . . . . . . . . . . . . . . . . . . . . . . . . . . . . . . . . 47

Figure S7 . . . . . . . . . . . . . . . . . . . . . . . . . . . . . . . . . 8

Figure S8 . . . . . . . . . . . . . . . . . . . . . . . . . . . . . . . . . . . . . . . . . . . 9

$\begin{array}{ll}\text { Supplementary Notes } & 10\end{array}$

Note S1 Model Non-dimensionalisation . . . . . . . . . . . . . . . . . . . . . . 10

Note S2 Model Implementation . . . . . . . . . . . . . . . . . . . . . . . . . 11

Note S3 Parameter Values. . . . . . . . . . . . . . . . . . . . . . . . . . . 12

Note S4 Killing Curves . . . . . . . . . . . . . . . . . . . . . . . . . . . . 13

Note S5 Cell Survival when $\gamma<\gamma_{c} . \quad$. . . . . . . . . . . . . . . . . . . . . . . . . . . . . 15

Note S6 Cell Survival when $\gamma<\gamma_{c}(t)$. . . . . . . . . . . . . . . . . . . . . . . . . . . . . . . 16

Note S7 A Discontinuous Adaptive Process . . . . . . . . . . . . . . . . . . . . 18

Note S8 . . . . . . . . . . . . . . . . . . . . . . . . . . . . . . . . . 20

Note S9 . . . . . . . . . . . . . . . . . . . . . . . . . . . . . . . . . . 20

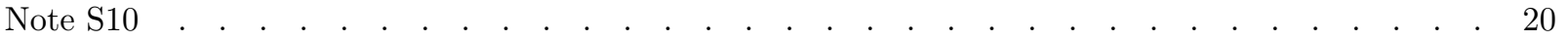

Note S11 . . . . . . . . . . . . . . . . . . . . . . . . . . . . . . . . . 20

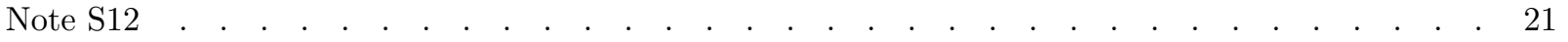

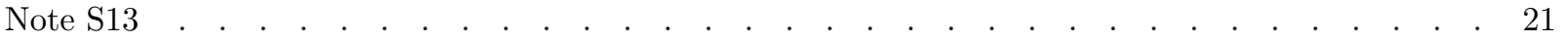

Note S14 . . . . . . . . . . . . . . . . . . . . . . . . . . . . . . . . . 21

Note S15 . . . . . . . . . . . . . . . . . . . . . . . . . . . . . . . . . 22

Note $\mathrm{S} 16$. . . . . . . . . . . . . . . . . . . . . . . . . . . . . . . 22

Note S17 . . . . . . . . . . . . . . . . . . . . . . . . . . . . . . . . . 22

Note S18 . . . . . . . . . . . . . . . . . . . . . . . . . . . . . . . . . 22

Note S19 . . . . . . . . . . . . . . . . . . . . . . . . . . . . . . . . . 23

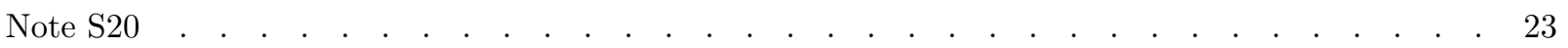

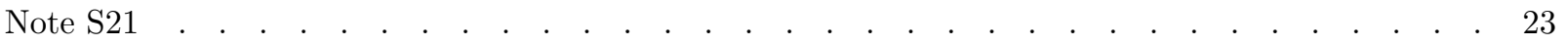

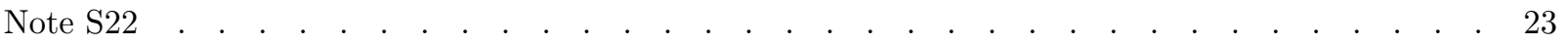

Note S23 . . . . . . . . . . . . . . . . . . . . . . . . . . . . . . . . . 24

Note S24 . . . . . . . . . . . . . . . . . . . . . . . . . . . . . . . . . 24

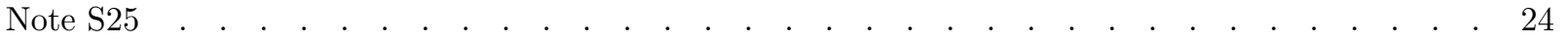

Note S26 . . . . . . . . . . . . . . . . . . . . . . . . . . . . . . . . . 24

Note S27 . . . . . . . . . . . . . . . . . . . . . . . . . . . . . . . . . 24

Note S28 . . . . . . . . . . . . . . . . . . . . . . . . . . . . . . . . . 24

Note S29 . . . . . . . . . . . . . . . . . . . . . . . . . . . . . . . . . . . . . . $\quad . \quad 25$

Note $\mathrm{S} 30$. . . . . . . . . . . . . . . . . . . . . . . . . . . . . . . . . 25

$\begin{array}{ll}\text { Supplementary References } & 26\end{array}$ 


\section{Supplementary Figures}

\section{Figure S1}
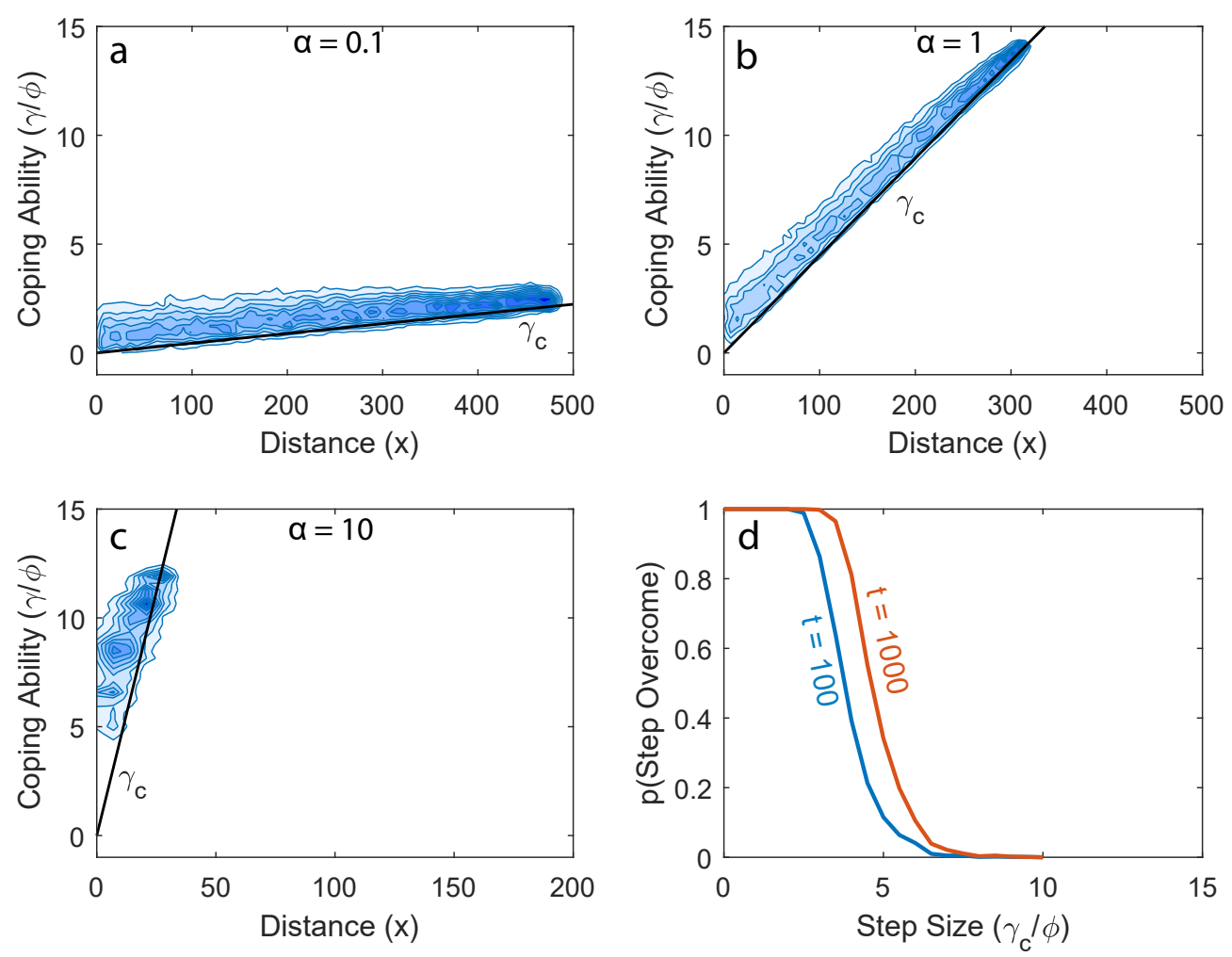

Figure S1: a,b,c) Probability density level curves for a population of cells adapting to a spatial antibiotic ramp $\gamma_{c}=\alpha x$ with various values of $\alpha$. Each simulation is run until $t=200$ units. The speed of adaptation $(d \gamma / d t)$ is plot in Fig. 2b. d) The probability of a step in antibiotic being overcome within a given time as a function of step height $\gamma_{c}$. This simulation is described in Note S10. 


\section{Figure S2}
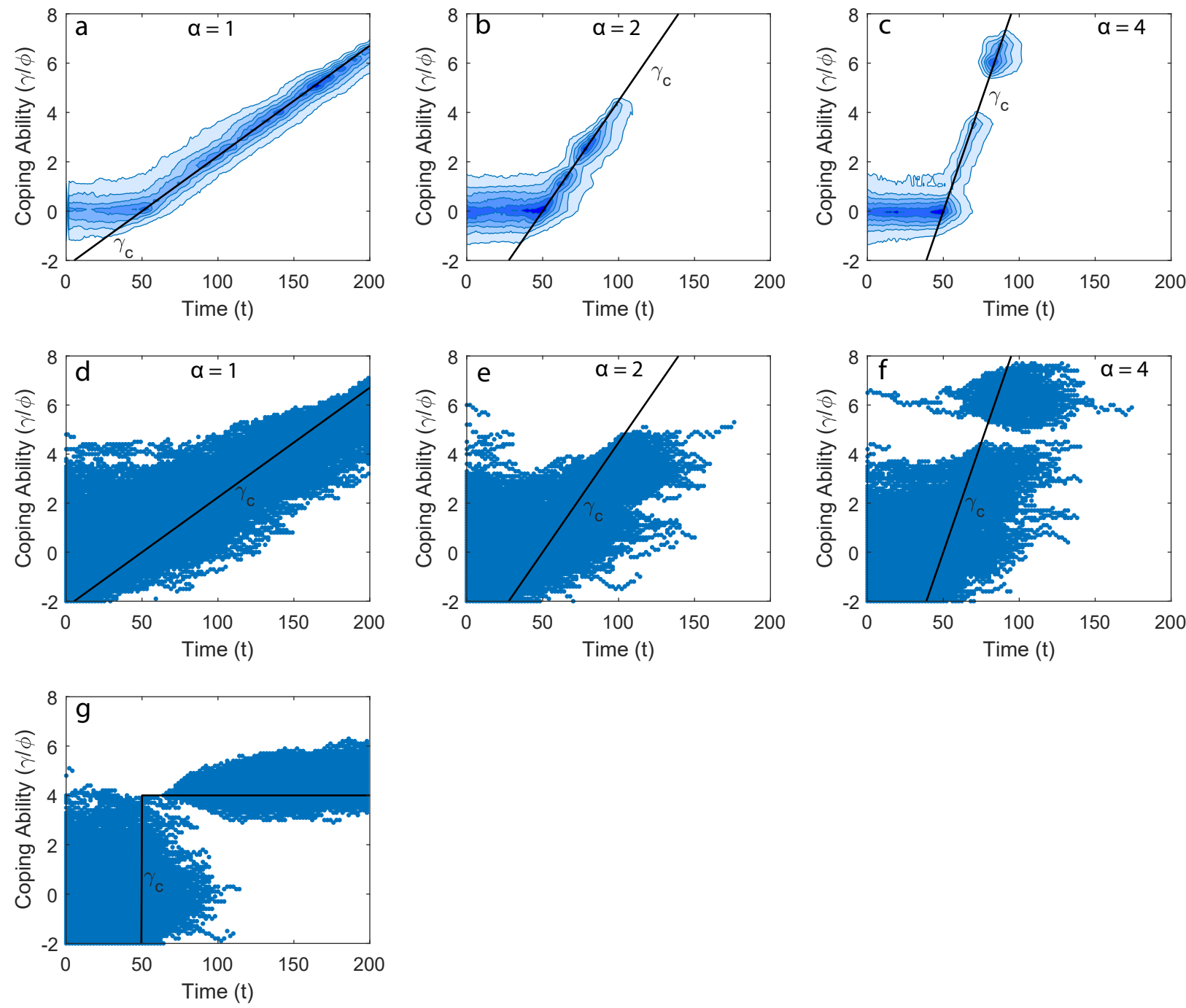

Figure S2: $\mathbf{a}, \mathbf{b}, \mathbf{c})$ Probability density level curves for a population of cells adapting to a temporal antibiotic ramp $\gamma_{c}=\alpha(t-50)$ with various values of $\alpha$. The probability of success in each case is plot in Fig. 3b: For $\alpha>1$ the population is unable to adapt sufficiently quickly and will die out. d,e,f) Individual cells plot for the simulations in $\mathbf{a}, \mathbf{b}, \mathbf{c}$. We observe that single cell lines that begin with high $\gamma$ can keep the population alive for a while but again, ultimately die out. g) A population of cells adapting to a step rise in antibiotic concentration which goes from $\gamma_{c}=-\infty$ to $\gamma_{c}=4 \phi$ at $t=50$, similar to Fig. 3c. Despite all cells having $\gamma<\gamma_{c}$ at $t=50$ the population survives as one cell with $\gamma$ just below $\gamma_{c}$ is able to evolve to have $\gamma>\gamma_{c}$ before dying. 


\section{Figure S3}

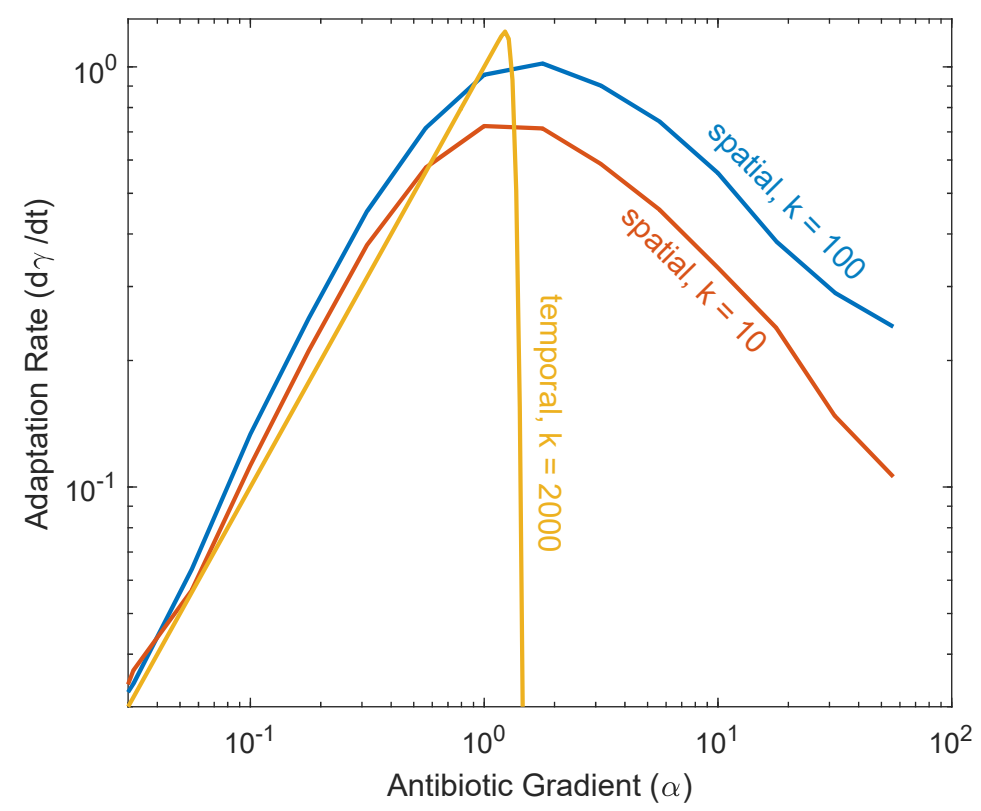

Figure S3: Comparison of adaptation rate for spatial and temporal antibiotic gradients $\alpha$. Spatial adaptation rates are as in Fig. 2b of the main text. Temporal adaptation rates are calculated by multiplying the probability of population survival (Fig. 3b of the main text) by the gradient $\alpha$. The adaptation rate for temporal simulations is thus equal to $\alpha$ up until a critical value (here $\alpha \approx 1$ ) at which point the population is not able to mutate sufficiently quickly to keep up with the antibiotic increase. 


\section{Figure S4}

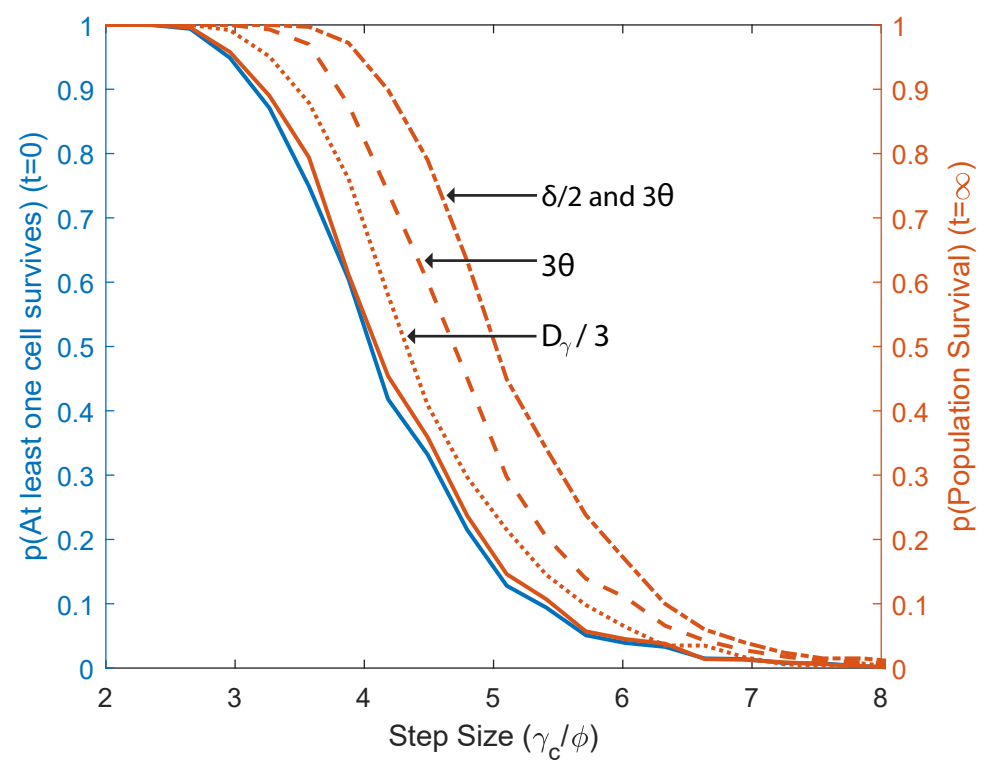

Figure S4: Plot similar to Fig. 3d demonstrating the probability of a step rise in antibiotic concentration being overcome by a population of cells. See Note S14 for detailed description of the simulation performed. In each case we vary the simulation parameters, and observe the changed probability of population survival. Note that here we plot (on the left axis) the probability of at least one cell surviving (out of a population of 2000), whereas in Fig. 3d we plot the probability of survival for a random (single) cell. The probability of at least one cell surviving is the same in each case, since $\phi$ (which defines the height of the antibiotic step) is varied as system parameters change. The difference between the probability that at least one cell survives, and the probability that the population as a whole survives, is a result of cells with $\gamma<\gamma_{c}$ which then evolve to have $\gamma>\gamma_{c}$ prior to dying. This situation is analysed analytically in Note S5. 


\section{Figure S5}
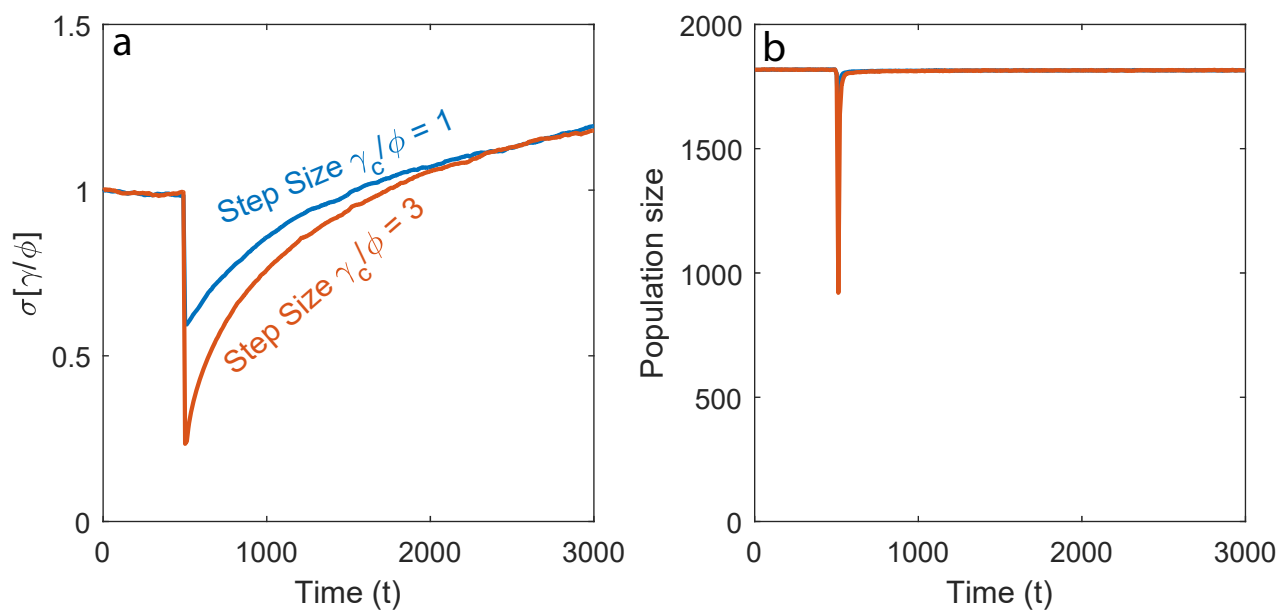

Figure S5: a) Standard deviation of $\gamma$ values in a population after it is subjected to a step of varying height at $t=500$ (note the calculation of $\sigma$ only considers cells with $\gamma>\gamma_{c}$, unlike Fig. 3e which considers all cells). We observe a rapid drop in $\sigma$ following the antibiotic step (as cells with low $\gamma$ are immediately eliminated from the calculation), but the population recovers its genetic diversity slowly over time. b) Population size over time, demonstrating that the population rapidly recovers (in terms of number of cells) following antibiotic dosing (Note S16). 


\section{Figure S6}
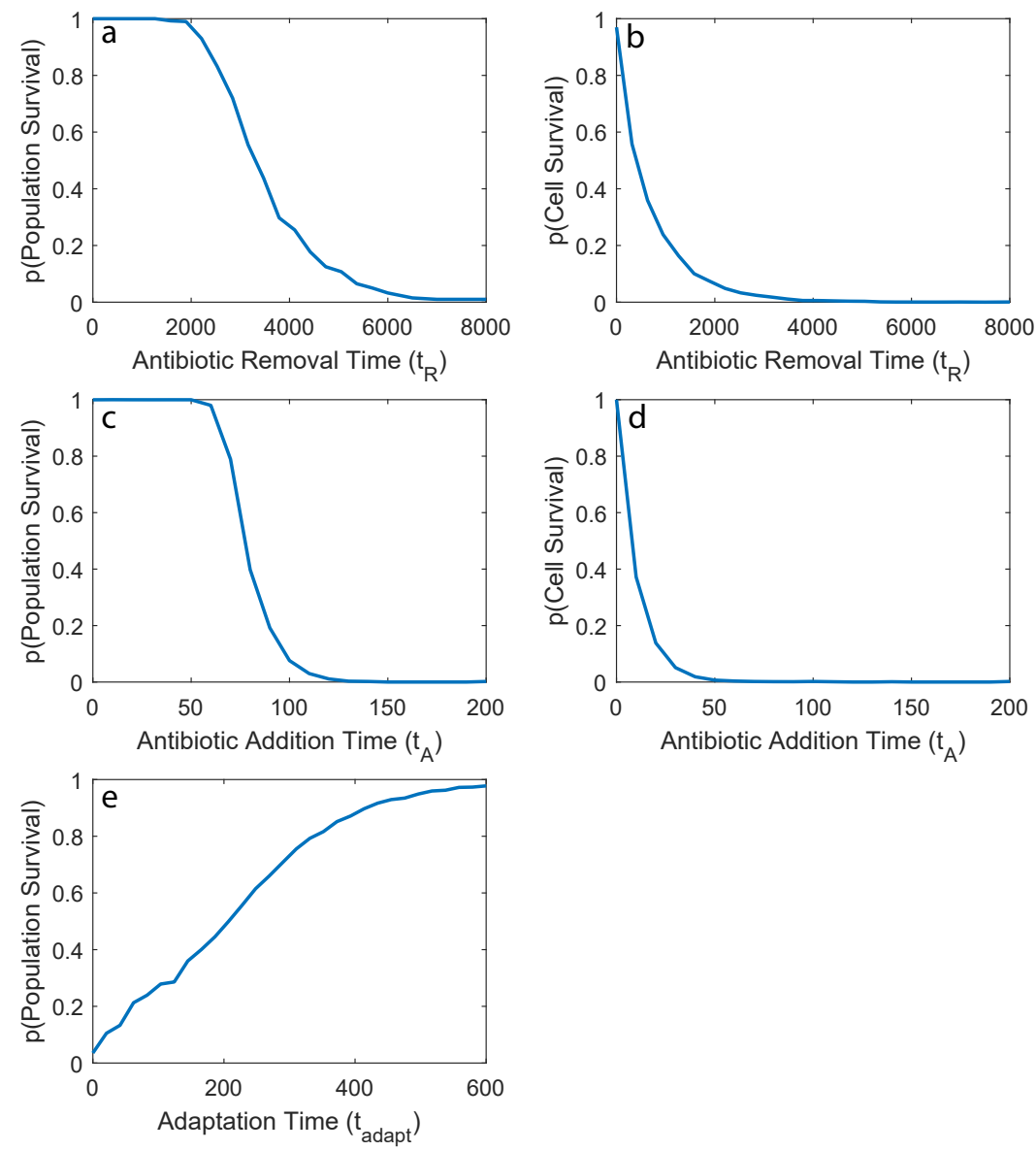

Figure S6: a,b) The probability of an already resistant population (a) or random cell (b) surviving readdition of antibiotic following its removal for a period of length $t_{R}$, as described in Note S18. When $t_{R}$ is large there is sufficient time for the population's $\gamma$ value to decay (due to $\theta$ returning it to the wildtype equilibrium), and thus the population may not survive when antibiotic is re-added after $t_{R}$. c,d) The probability of a population (c) or a random cell (d) surviving addition of antibiotic for $t_{A}$ and its subsequent removal, as described in Note S19. When $t_{A}$ is large there is sufficient time for the population to die out (with this time-scale determined by $\delta$ ), and thus there may be no surviving cells when antibiotic is removed at $t_{A}$. Here we observe that the population decay in $\mathbf{a}, \mathbf{b}$ occurs over a time scale that is approximately two orders of magnitude longer than that in $\mathbf{c}, \mathbf{d}$. The relative length of these time-scales is determined by the relative sizes of $\theta$ and $\delta$ respectively. e) The probability of a population of cells surviving two sequential step rises in antibiotic concentration as a function of how long is spent adapting at the intermediate antibiotic concentration, $t_{\text {adapt }}$ (Note S20). 


\section{Figure S7}
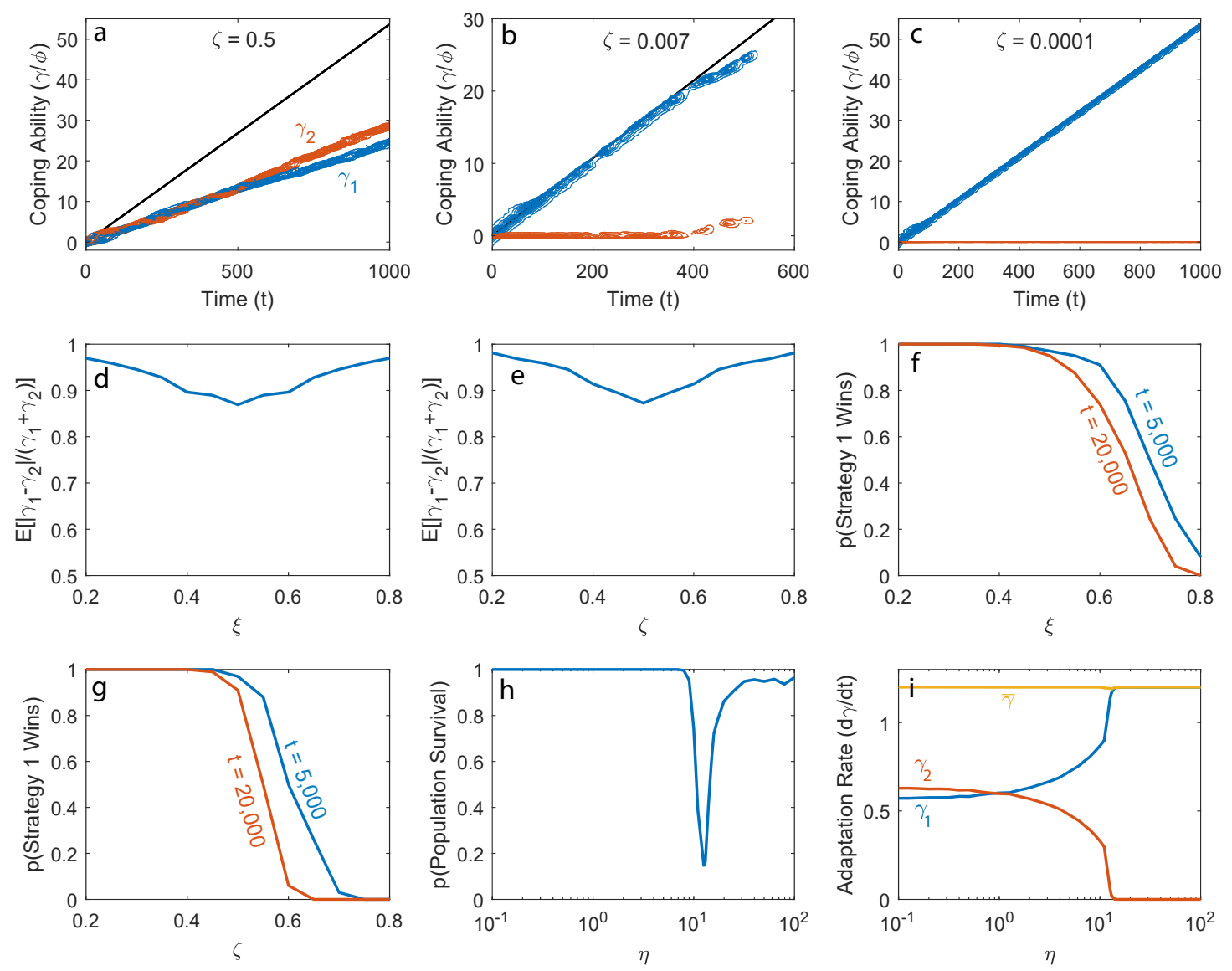

Figure S7: a,b,c) Probability density level curves for a population of cells with two available resistance strategies adapting to a temporal antibiotic ramp $\gamma_{c}=\alpha t$ with $\alpha=1.2$ for various $\zeta$ values (Note S21). When $\zeta$ is large both strategies develop (see Fig. 4b), whilst for small $\zeta$ only $\gamma_{1}$ does. For intermediate values $\zeta \approx 0.01$ there is a significant probability that the population will die out, as illustrated in Fig. 4a. d,e) The expected magnitude of the difference between mean coping strategies within a cell population $\left(\left|\gamma_{1}-\gamma_{2}\right|\right)$ normalised by the total coping ability $\left(\gamma_{1}+\gamma_{2}=\bar{\gamma}\right)$, (Note S22). This factor provides a measure of dominance of a single strategy: If only one develops (e.g. $\gamma_{2} \approx 0$ so that $\gamma_{1} \approx \bar{\gamma}$ ) then it is equal to $\sim 1$, whereas if both strategies develop evenly $\left(\gamma_{1} \approx \gamma_{2} \approx \bar{\gamma} / 2\right)$ then it is $\sim 0$. This value is typically near unity for our simulations, implying that $\bar{\gamma}$ is predominantly determined by a single resistance strategy. f,g) Plots similar to Fig. 4d but with simulations initialised with $E\left[\gamma_{1}\right]=4 \phi$ (Note S23). We observe that even if $\gamma_{1}$ is initially favoured, if $\gamma_{2}$ is "better" (large $\xi$ or small $\zeta$ ) then it can overcome $\gamma_{1}$ 's early lead and take over the population. There is some probability of this occurring during each time window, and thus at small times the outcome is more likely to align with its initial state $\left(\gamma_{1}\right.$ being ahead). h,i) Similar to Fig. 4a,b of the main text, but with $\theta_{1}=\theta$ and $\theta_{2}=\eta \theta$ (Note S24). In $\mathbf{h}$ we observe a region of reduced probability of population survival arising at the same ratio of theta values (i.e. $\theta_{1} / \theta_{2}$ ) to the dip observed in Fig. 4a. 


\section{Figure S8}
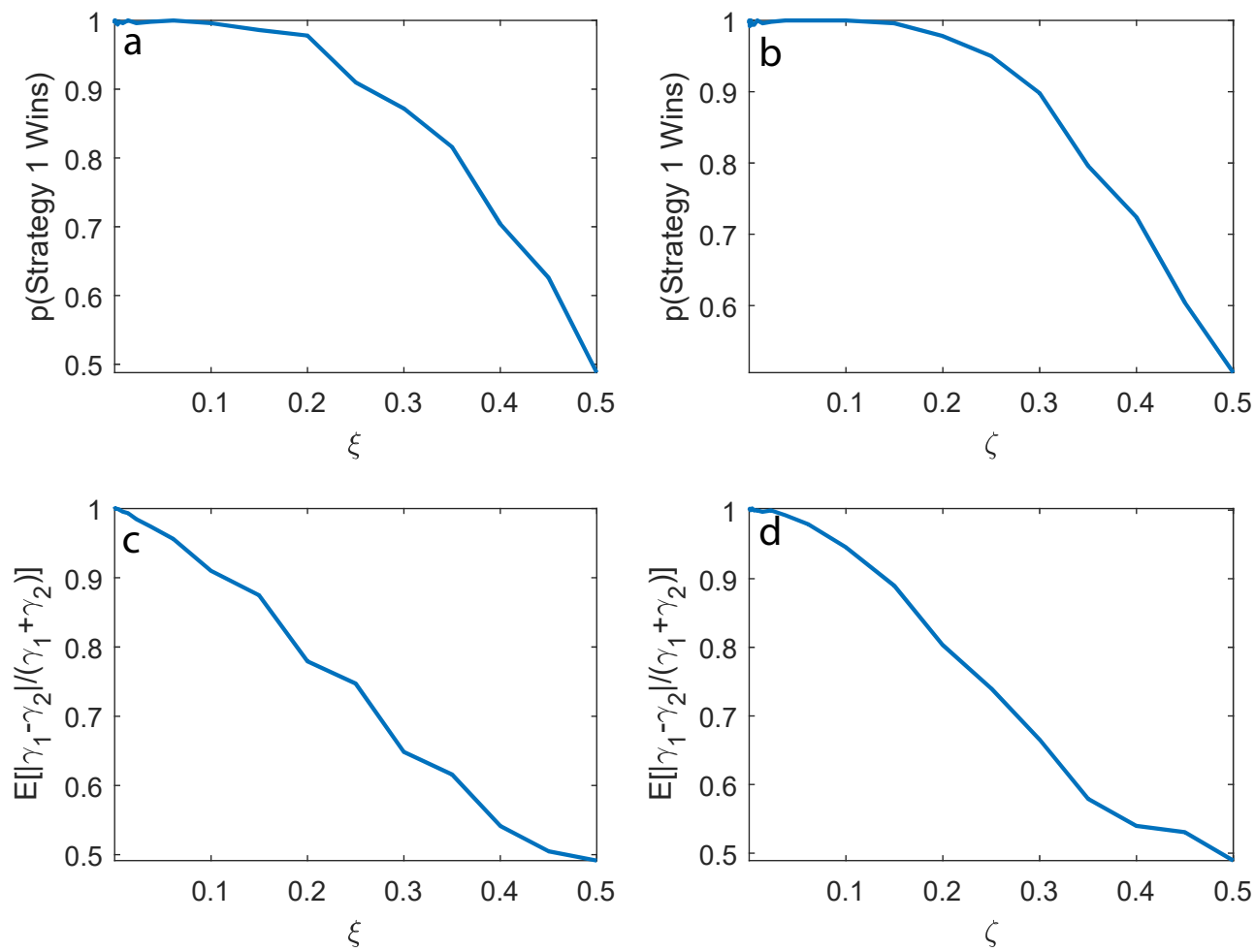

Figure S8: The behaviour of a population of cells with two coping strategies that encounter a spatial step in antibiotic concentration (Note S26). a,b) The probability that Strategy 1 wins (defined in Note S26) as a function of $\xi$ and $\zeta$. Results are qualitatively similar to the case of a temporal step (Fig. 4d). c,d) Quantification of dominance of a single strategy (similar to Fig. S7d,e) demonstrating that in this case the spatial step is more likely to encourage both strategies to develop to some degree (rather than one evolving to the exclusion of the other). 


\section{Supplementary Notes}

\section{Note S1 Model Non-dimensionalisation}

Following the results of Hermsen [1] we employ a dimensional form of Eq. (4) given by:

$$
\hat{R}_{i}=\hat{r}\left(1-\sum_{j} \frac{\Gamma\left(\hat{x}_{i}-\hat{x}_{j} \mid \sigma\right)}{\hat{k}}\right) H\left(\hat{\gamma}_{i}-\hat{\gamma}_{c}\left(\hat{\gamma}_{i}, \hat{t}\right)\right)
$$

Where $\hat{r}$ is growth rate, and parameters with a hat $\left({ }^{\wedge}\right)$ are dimensional versions of those used in the main text. Additionally, the dimensional form of Eq. (2) is given by:

$$
\mathrm{d} \hat{\gamma}_{t}=-\hat{\theta} \operatorname{sign}\left(\hat{\gamma}_{t}-\hat{\mu}\right) \mathrm{d} t+\sqrt{2 D_{\gamma} \hat{D}_{\gamma}} \mathrm{d} B_{t}
$$

and Eq. (3) by:

$$
\mathrm{d} \hat{x_{t}}=\sqrt{2 D_{x} \hat{D}_{x}} \mathrm{~d} B_{t}
$$

We non-dimensionalise these equations (dimensionless parameters used in the main text are listed in Table S1) by scaling $\hat{t}$ by the maximum reproduction rate when $\hat{\gamma}_{i}>\hat{\gamma}_{c}$, giving $t=(\hat{r}-\hat{\delta}) \hat{t}$. $\hat{x}$ and $\hat{\gamma}$ are scaled similarly, giving $x=\hat{x} \sqrt{(\hat{r}-\hat{\delta}) / \hat{D}_{x}}$ and $\gamma=\hat{\gamma} \sqrt{(\hat{r}-\hat{\delta}) / \hat{D}_{\gamma}}$ respectively. This allows us to substitute $\delta=\hat{\delta} /(\hat{r}-\hat{\delta})$ and hence the $\hat{r}$ pre-factor in Eq. (S1) becomes $\hat{r} /(\hat{r}-\hat{\delta})=1+\delta=r$. Similarly the dimensional width of the gaussian over which spatial averaging occurs is scaled as $\sigma=\hat{\sigma} \sqrt{(\hat{r}-\hat{\delta}) / \hat{D}_{x}}$, spatial carrying capacity by $k=\hat{k} / \sqrt{(\hat{r}-\hat{\delta}) / \hat{D}_{x}}, \mu=\hat{\mu} \sqrt{(\hat{r}-\hat{\delta}) / \hat{D}_{\gamma}}$ and the drift rate by $\theta=\hat{\theta} \sqrt{(\hat{r}-\hat{\delta}) / \hat{D}_{\gamma}}$. This re-scaling means that the effective growth rate when $\hat{\gamma}_{i}>\hat{\gamma}_{c}$ is equal to unity $(\hat{r}-\hat{\delta}=1)$. These parameterisations are summarised in Table S1. 


\begin{tabular}{c|c}
$\begin{array}{c}\text { Original Parameter } \\
\text { ine } \hat{t}\end{array}$ & $\begin{array}{c}\text { Dimensionless Version } \\
t=(\hat{r}-\hat{\delta}) \hat{t}\end{array}$ \\
$\hat{x}$ & $x=\hat{x} \sqrt{(\hat{r}-\hat{\delta}) / \hat{D}_{x}}$ \\
$\hat{\gamma}$ & $\gamma=\hat{\gamma} \sqrt{(\hat{r}-\hat{\delta}) / \hat{D}_{\gamma}}$ \\
$\hat{\delta}$ & $\delta=\hat{\delta} /(\hat{r}-\hat{\delta})$ \\
$\hat{r}$ & $r=1+\delta$ \\
$\hat{\sigma}$ & $\begin{array}{c}\sigma=\hat{\sigma} \sqrt{(\hat{r}-\hat{\delta}) / \hat{D}_{x}} \\
\hat{k} \\
\hat{\mu}\end{array} \quad \hat{k} / \sqrt{(\hat{r}-\hat{\delta}) / \hat{D}_{x}}$ \\
$\hat{\theta}$ & $\theta=\hat{\mu} \sqrt{(\hat{r}-\hat{\delta}) / \hat{D}_{\gamma}}$ \\
& $\theta=\hat{\theta} \sqrt{(\hat{r}-\hat{\delta}) / \hat{D}_{\gamma}}$
\end{tabular}

Table S1: Parameters and dimensionless alternatives.

\section{Note S2 Model Implementation}

All simulations are implemented and performed in MATLAB 2017b with parameter values as described in Note S3. Simulations are typically initialised with $k$ cells (placed at $x=1$ ), and with $\gamma$ values sampled from a Laplace distribution with mean $\mu$ and standard deviation $\phi$. The system is then integrated numerically with a small timestep $\Delta$. This represents a "tau leaping" variant of the Gillespie algorithm [2], where we allow many reactions to occur within a single time step $\Delta$. This drastically reduces the computational cost of simulation when compared to approaches that model reactions (potentially for a very large number of cells) as occurring sequentially.

For simulations of spatial antibiotic profiles the most expensive computational task is the calculation of local cellular density in the function $\Gamma$ in Eq. (4). To reduce this cost drastically we evaluate $\Gamma$ at integer values of $x$ in each simulation loop, rather than at the location of each cell (thereby reducing the computation cost by a factor of $\approx k$ for spatial simulations). The spatial density at any given $x_{i}$ is then approximated as being equal to that at the nearest integer spatial location (i.e. round $\left(x_{i}\right)$ ). We assessed the validity of this approximation by comparing simulation results to those in which $\Gamma$ was evaluated at every $x_{i}$, observing no significant differences. For simulations with temporal antibiotic gradients (and $\sigma=\infty$ as in Table S2) we do not need to evaluate $\Gamma$, as it is equal to the total number of cells in the system at a given time.

The probabilities of reproduction or death within a time period $\Delta$ are given by $1-\exp \left(-R_{i} \Delta\right)$ and $1-\exp (-\delta \Delta)$ respectively. Diffusion terms $\left(\mathrm{d} B_{t}\right.$ in Eq. (2) and Eq. (3)) are simulated by drawing samples from a normal distribution with mean 0 and standard deviations of $\sqrt{2 D_{x} \Delta}$ and $\sqrt{2 D_{\gamma} \Delta}$ respectively. Spatial boundaries (i.e. $x=0$ ) are implemented by setting any cell with location $x<0$ to $x=0$ at the end of each simulation loop.

Additional implementation details for specific simulations are described the subsequent Supplementary Notes. 


\section{Note S3 Parameter Values}

Following from Note S1, the remaining free parameters for the model (when only one resistance strategy is considered) are $\delta, k, \mu, \theta$, and $\sigma$. The values used in all simulations (unless otherwise specified) are listed in Table S2. Different values for $k$ and $\sigma$ are used when simulating temporal versus spatial antibiotic gradients. When a temporal gradient is considered it is assumed that the system is well mixed, thus resources are shared between all cells (hence $\sigma=\infty$ ). We increase $k$ in this case so that simulations give more statistically-reliable outcome (i.e. an average over a larger number of cells).

\begin{tabular}{c|c} 
Parameter & Value \\
ine $\delta$ & 0.1 \\
$\mu$ & 0 \\
$\theta$ & 0.0632 \\
$D_{x}$ & 1 \\
$D_{\gamma}$ & 1 \\
$k$ (spatial antibiotic gradient) & 100 \\
$k$ (temporal antibiotic gradient) & 2000 \\
$\sigma$ (spatial antibiotic gradient) & 1 \\
$\sigma$ (temporal antibiotic gradient) & $\infty$
\end{tabular}

Table S2: Parameters values used for spatial and temporal simulations.

The above parameters yield a value $\phi=\sqrt{2} D_{\gamma} / \theta=22.36$ where $\phi$ is the standard deviation of the stable distribution of Eq. (2) when no selection occurs.

When we consider multiple strategies for resistance we maintain the parameter values in Table S2, except for where we vary these according to the parameters $\zeta$ and $\xi$. We therefore have $\mu_{1}=\mu_{2}=\mu=0$ throughout. 


\section{Note S4 Killing Curves}

In Fig. 3d of the main text we plot a concentration killing curve (CKC) [3] as a function of $\gamma_{c}$. This killing curve follows analytically from the Laplace distribution expected to govern $\gamma$ when cells are not under antibiotic selection. The CDF of a Laplace distribution can be expressed as:

$$
1-C D F(\gamma)=C(\gamma)= \begin{cases}1-\frac{1}{2} \exp \left(\frac{\gamma-\mu}{b}\right) & \gamma \in[0, \mu] \\ \frac{1}{2} \exp \left(-\frac{\gamma-\mu}{b}\right) & \gamma \in[\mu, \infty)\end{cases}
$$

If we substitute $\gamma-\mu=\log \left([A] /[A]_{50}\right)$ (where we are normalising concentration by $[A]_{50}$ ) as in Eq. (1) we get:

$$
C([A])= \begin{cases}1-\frac{1}{2}\left([A] /[A]_{50}\right)^{1 / b} & {[A] \in\left[0,[A]_{50}\right]} \\ \frac{1}{2}\left([A] /[A]_{50}\right)^{-1 / b} & {[A] \in\left[[A]_{50}, \infty\right)}\end{cases}
$$

Killing curves used to fit experimental data [3] can take the form of:

$$
N([A])=\frac{1}{1+\exp \left(r\left([A]-[A]_{50}\right)\right.}
$$

Which (given aligned parameter choices) provides a visually similar curve, as illustrated in Fig. S9. One significant difference between these distributions is that $C$ has a power-law tail (linear in the log-log plot in Fig. S9b), whilst $\mathrm{N}$ has an exponential tail. $C$ will therefore tend to have a "heavier" tail because:

$$
\lim _{[A] \rightarrow \infty} \frac{N([A])}{C([A])}=0
$$
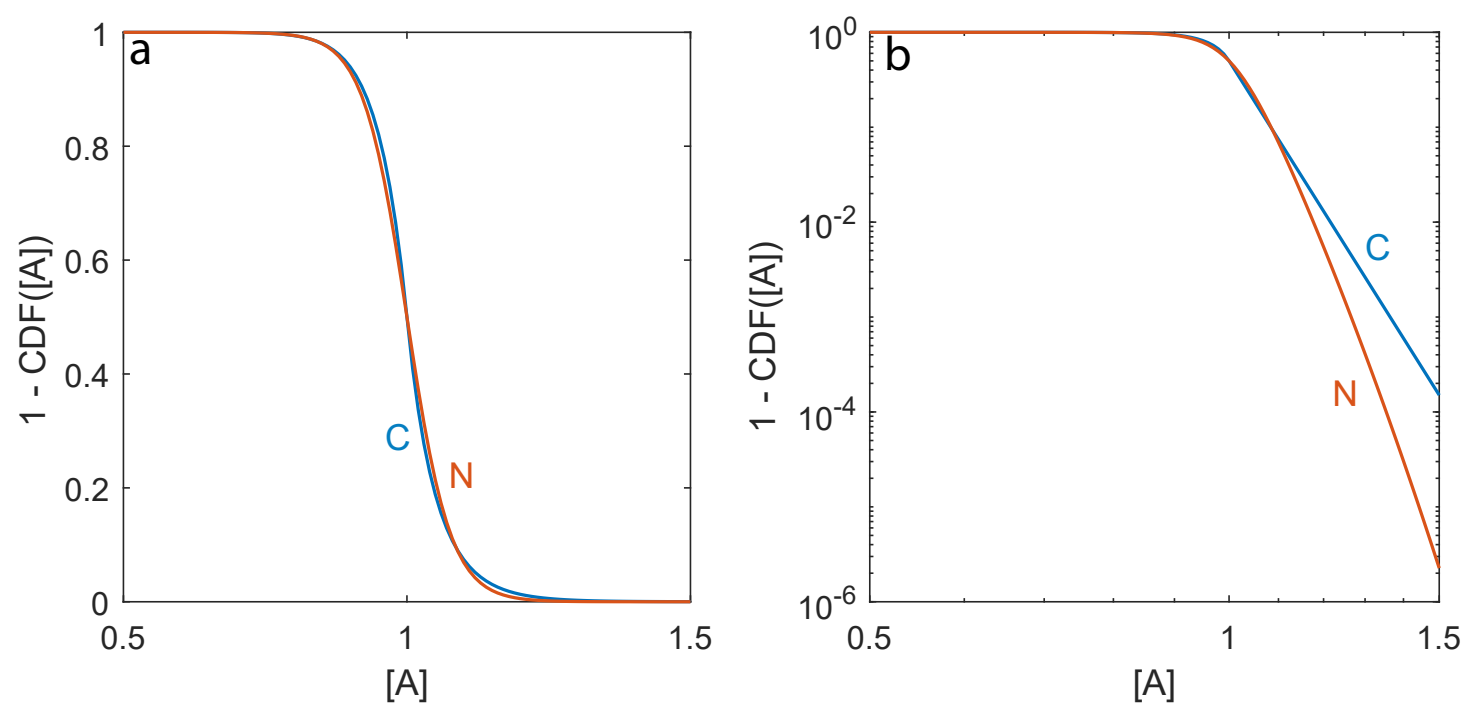

Figure S9: Examples of the functions Eq. (S5) and Eq. (S6) with $b=0.05, r=26$, and $[A]_{50}=1$. $\mathbf{b}$ is the same data as a plot on log-scale.

Another interesting question regards what our model predicts the shape of CKC to be following a period of adaptation to an intermediate antibiotic concentration. In this case the distribution of $\gamma$ changes due to selection (cells with $\gamma<\gamma_{c}$ do not replicate) as illustrated in Fig. 1. In Fig. S10a we plot examples of killing curves for a wild-type population of cells $C([A])$, and for a population that has spent a long period adapting to $\gamma_{c}=0$ (i.e. antibiotic concentration $\left.[A]_{50}\right)$ with $\mu=-\infty, C_{2}([A])$. The wild-type curve is symmetric about $\gamma_{c}=0$, but almost all of the adapted cells have $\gamma_{c}>0$. The adapted distribution appears to have an approximately exponential tail (Fig. S10b) near to $[A]=1$, though it may approach the power-law tail of $C$ 
for very large $[A]$. The adapted distributions shape could be made more symmetric (closer to the sigmoidal wild-type shape) if a "soft" killing criterion for cells with $\gamma<\gamma_{c}$ were used. That is, if the reproduction rate in Eq. (4) varied smoothly when $\gamma$ was near to $\gamma_{c}$, rather than the sharp heaviside step function used at present.
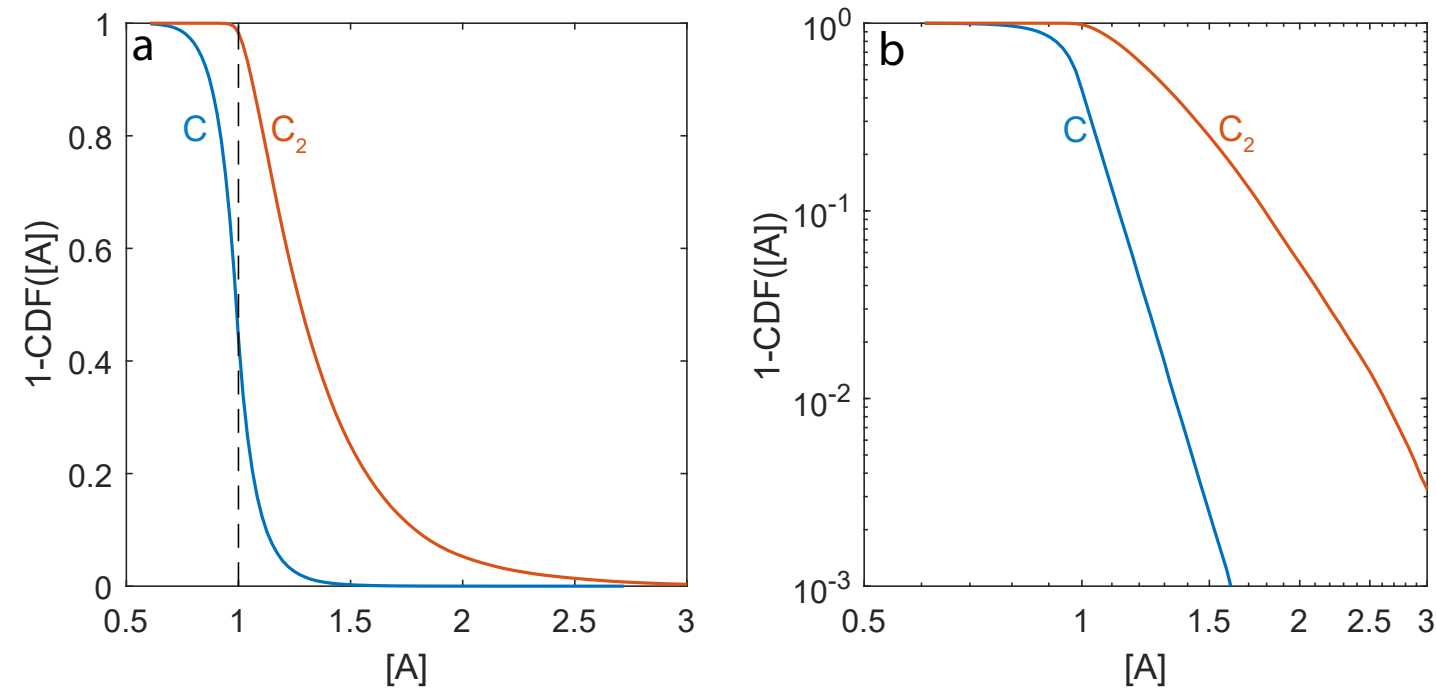

Figure S10: Examples of steady-state CKCs (again with $[A]_{50}=1$ ) for cells that are not undergoing selection for antibiotic resistance $(C)$, and after undergoing a selective growth at $[A]=1\left(C_{2}\right)$. b is the same data as a plot on log-scale. 


\section{Note S5 Cell Survival when $\gamma<\gamma_{c}$}

In this section we derive an expression for the approximate probability that a cell with $\gamma<\gamma_{c}$ will reach $\gamma_{c}$ before dying following a step rise in antibiotic.

We assume that the influence of the drift term (governed by $\theta$ ) upon the short term behaviour of Eq. (2) is small, which allows us to treat the evolution of $\gamma$ as a pure brownian motion. Further, we assume that any particle that reaches $\gamma_{c}$ will rapidly replicate, and thus this $\left(\gamma\right.$ reaching $\left.\gamma_{c}\right)$ is the criteria for population survival. Finally, for a fixed step height that is proportional to $\phi$ (i.e. $\gamma_{c} \propto \phi$ ) we have that both the width of the distribution of $\gamma$ values and $\gamma_{c}$ are scaled by $\phi$, and as such we set $\gamma_{c}-\gamma=\nu \phi$ for an arbitrarily proportionality constant $\nu$.

The First Passage Time Density (FPTD) is the probability that a cell starting at $\gamma$ first reaches $\gamma_{c}$ at time $t$, which for perfectly brownian motion is given by:

$$
\operatorname{FPTD}(t)=\frac{\left|\gamma_{c}-\gamma\right|}{\sqrt{4 \pi D_{\gamma} t^{3}}} \exp \left(-\frac{\left(\gamma_{c}-\gamma\right)^{2}}{4 D_{\gamma} t}\right)
$$

which substituting $\gamma_{c}-\gamma=\nu \phi=\nu \sqrt{2} D_{\gamma} / \theta$ is equal to:

$$
\operatorname{FPTD}(t)=\frac{\nu \sqrt{D_{\gamma}}}{\theta \sqrt{2 \pi t^{3}}} \exp \left(-\frac{\nu^{2} D_{\gamma}}{2 \theta^{2} t}\right)
$$

Now, given the cell death rate when $\gamma<\gamma_{c}$ is equal to $\delta$, the probability of a cell surviving $(S)$ from time 0 until $t$ is:

$$
S(t)=\exp (-\delta t)
$$

Therefore, the probability that a cell reaches $\gamma_{c}$ at time $t$ without dying is the probability that the cell is alive at this point multiplied by the probability of reaching $\gamma_{c}, F P T D(t) \times S(t)$. The overall probability that a cell reaches $\gamma_{c}$ prior to dying is thus given by:

$$
p(\text { PopulationSurvival })=\int_{0}^{\infty} S(t) \times F \operatorname{PTD}(t) d t=\int_{0}^{\infty} \exp (-\delta t) \times \frac{\nu \sqrt{D_{\gamma}}}{\theta \sqrt{2 \pi t^{3}}} \exp \left(-\frac{\nu^{2} D_{\gamma}}{2 \theta^{2} t}\right) d t
$$

which we evaluate to give:

$$
p(\text { Population Survival })=\exp \left(-\frac{\nu \sqrt{2 D_{\gamma} \delta}}{\theta}\right)
$$

Note that the dependence of this result upon $\theta$ does not contradict our initial assumption that the influence of drift due to $\theta$ would not significantly impact this probability. This is because in Eq. (S12) $\theta$ is introduced due to its impact on the steady-state distribution of $\gamma$ values prior to introduction of antibiotic, rather than because of its influence on the short-term dynamics of $\gamma$ following antibiotic addition. Similarly, it is worth noting that larger values of $D_{\gamma}$ in Eq. (S12) lead to a lower probability of survival since they also determine the initial distribution of $\gamma$ values. To highlight this we can alternatively express Eq. (S12) in terms of the absolute difference in initial $\gamma$ values, $\beta=\gamma_{c}-\gamma$, which gives:

$$
p(\text { Population Survival })=\exp \left(-\beta \sqrt{\frac{\delta}{D_{\gamma}}}\right)
$$




\section{Note S6 Cell Survival when $\gamma<\gamma_{c}(t)$}

We can extend the analysis in Note S5 to consider a case in which $\gamma_{c}$ has a linear dependence on time, given by:

$$
\gamma_{c}(t)=\beta+\alpha t
$$

where $\beta=\gamma_{c}-\gamma$ is the difference between a cell's starting $\gamma$ value and the height of the step encountered $\left(\gamma_{c}\right)$ at $t=0$ (as defined in Note S5). $\alpha$ is the temporal gradient in antibiotic concentration as defined in the main text. To analytically investigate the impact of this antibiotic gradient we can use the result [4]:

$$
P\left(\sup _{0 \leq T \leq t}(\gamma(T)-\alpha T) \geq \beta\right)=1-f_{1}\left(\frac{\beta}{\sqrt{2 D_{\gamma} t}}+\alpha \sqrt{2 D_{\gamma} t}\right)+\exp (-2 \alpha \beta) f_{1}\left(\frac{-\beta}{\sqrt{2 D_{\gamma} t}}+\alpha \sqrt{2 D_{\gamma} t}\right)
$$

where $f_{1}$ is the $\mathrm{CDF}$ of a standard normal distribution. This result (which can be derived using martingale theory [5]) describes the probability that a cell initially with $\gamma<\gamma_{c}$ reaches $\gamma_{c}$ during the interval $0 \leq T \leq t$. This expression can be linked to the FPTD using:

$$
F P T D(t)=\frac{\partial P\left(\sup _{0 \leq T \leq t}(\gamma(T)-\alpha T) \geq \beta\right)}{\partial t}
$$

which yields

$$
F P T D(t)=\frac{\nu \sqrt{D_{\gamma}}}{\theta \sqrt{2 \pi t^{3}}} \exp \left(-\frac{\nu^{2} D_{\gamma}}{2 \theta^{2} t}\right) \times \exp \left(-\alpha^{2} D_{\gamma} t\right) \times \exp \left(-\frac{\alpha \nu D_{\gamma} \sqrt{2}}{\theta}\right)
$$

We note that the first term in Eq. (S17) is the same as Eq. (S9), the second term is similar in form to the cell death term $S(t)$ from Eq. (S10), and the third term is independent of time. It is therefore straightforward to perform the integral as in Eq. (S11) which yields:

$$
p(\text { Population Survival })=\exp \left(-\beta\left(\alpha+\sqrt{\alpha^{2}+\delta / D_{\gamma}}\right)\right)
$$

which if we substitute $\beta=\nu \sqrt{2} D_{\gamma} / \theta$ gives:

$$
p(\text { Population Survival })=\exp \left(-\frac{\nu \sqrt{2} D_{\gamma}}{\theta}\left(\alpha+\sqrt{\alpha^{2}+\delta / D_{\gamma}}\right)\right)
$$

We observe that if $\alpha=0$ then Eq. (S19) is equivalent to Eq. (S12). Furthermore, when $\alpha>>\delta / D_{\gamma}$ we have that:

$$
p(\text { Population Survival }) \propto \exp (-\alpha)
$$

indicating that the probability of a cell's survival decreases exponentially with a large temporal gradient $\alpha$.

In Fig. S11 we plot the behaviour of Eq. (S19) as both $\nu$ and $\alpha$ are varied in order to investigate the impact of a temporal gradient $\alpha$ on a cell's survival probability. We observe that the presence of a positive $(\alpha>0)$ temporal gradient in antibiotic concentration leads to a rapid reduction in the cell's survival probability, and that (as expected) cells starting with $\gamma$ near the step height of the step in $\gamma_{c}$ (i.e. $\beta$ and consequently $\nu$ are small) are much more likely to catch up to $\gamma_{c}(t)$ prior to dying out. This analysis also makes clear the impact of a reduction in antibiotic following a step rise (i.e. $\alpha<0$, Fig. S11b): If insufficient time is spent at high antibiotic concentrations the pulse of antibiotic is overcome with high probability.

It is worth remarking that the analysis in this note makes significant additional assumptions when compared to the full stochastic model used in the main text. In particular, we have calculated the probability of a single cell's $\gamma$ exceeding $\gamma_{c}$ before it dies, however this does not necessarily guarantee that it is able to establish a stable population at this point. This limits our ability to apply the analytical results derived in this note to (for example) situations as in Fig. 3b, where there is a continuous birth-death process of cells with varying $\gamma$ as $\alpha$ increases. In that case there is a distribution of $\gamma$ values in the population at any time (e.g. as in Fig. S2), which is not reflected in the analysis in Fig. S11 (where a single cell with fixed initial $\gamma$ is considered). 

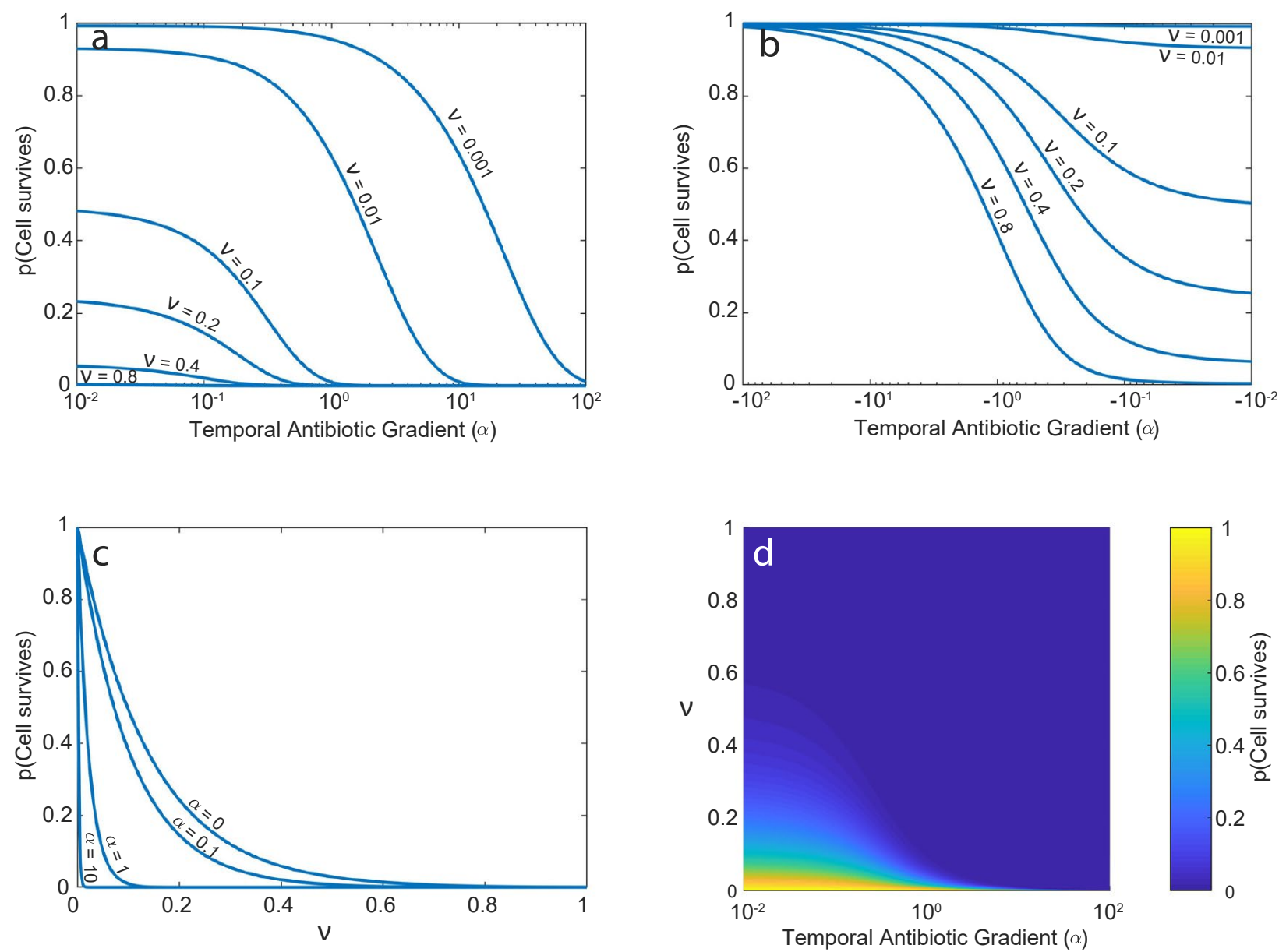

Figure S11: The probability of a single cell (with fixed initial $\gamma$ ) reaching an antibiotic level $\gamma_{c}(t)$ prior to dying. Cases where $\alpha=0$ correspond to a fixed-height step rise in antibiotic concentration. a) Probability of a cell's survival according to Eq. (S19) for various $\nu$ as a function of $\alpha$. (b) Similar to a, but for negative $\alpha$. (c) Probability of a cell's survival according to Eq. (S19) for various $\alpha$ as a function of $\nu$. (d) 2-D plot of dependence of cell survival probability on both $\alpha$ and $\nu$. 


\section{Note S7 A Discontinuous Adaptive Process}

In this section we outline an alternative form of Eq. (2) of the main text, which allows us to treat the evolutionary process as happening in discrete steps, rather than as a continuous process. This reflects the outcome of experimental studies which have found that in some cases resistance can develop in jumps due to distinct high-impact mutations $[6,7,8]$. We demonstrate that even when this is the case our simulations yield results approximately equivalent to those in the main text (where adaptive processes were continuous).

For the discrete evolutionary case, in which cells can only have $\gamma$ values that are discrete (integer) multiples of a fixed quantity $\omega$ we propose an equivalent to Eq. (2) of the form:

$$
\mathrm{d} \gamma_{t}=\omega\left(\mathrm{d} Z_{t}\left(\lambda_{1}\right)-\mathrm{d} Z_{t}\left(\lambda_{2}\right)-\operatorname{sign}\left(\gamma_{t}-\mu\right) \mathrm{d} Z_{t}\left(\lambda_{3}\right)\right)
$$

where $\mathrm{d} Z_{t}\left(\lambda_{i}\right)$ is a number sampled from a Poisson distribution with parameter $\lambda_{i}$. The first term in Eq. (S21) represents positive mutational steps (that increase antibiotic resistance), the second represents mutational steps that decrease resistance, and the third represents introduces a bias in this process toward the wild-type state $\mu$ as was present in Eq. (2). Eq. (S21) is an example of a jump stochastic differential equation [9], a lesser-studied kind of stochastic differential equation which is frequently applied for applications in financial mathematics. In order to achieve equivalence between the parameters in Eq. (2) and Eq. (S21) we can judiciously choose each $\lambda_{i}$. To do this we take advantage of the fact that the sum of two Poisson random variables with parameters $\lambda_{i}$ and $\lambda_{j}$ yields a Poisson random variable with parameter $\lambda_{i}+\lambda_{j}$, and that the difference between two Poisson random variables is distributed according to the Skellam distribution which has mean $\lambda_{i}-\lambda_{j}$ and variance $\lambda_{i}+\lambda_{j}$. Thus, we set:

$$
\begin{aligned}
\lambda_{1}=\lambda_{2} & =\frac{D_{\gamma} \mathrm{d} t}{\omega^{2}}-\frac{\theta \mathrm{d} t}{2 \omega} \\
\lambda_{3} & =\frac{\theta \mathrm{d} t}{\omega}
\end{aligned}
$$

where $\mathrm{d} t$ is the simulation timestep, and other system parameters are as defined in the main text. The mean (expected value) of a random step drawn from Eq. (S21) is therefore equal to:

$$
\mathbb{E}\left(\mathrm{d} \gamma_{t}\right)=\omega\left(\lambda_{1}-\lambda_{2}-\operatorname{sign}\left(\gamma_{t}-\mu\right) \frac{\theta \mathrm{d} t}{\omega}\right)=-\theta \operatorname{sign}\left(\gamma_{t}-\mu\right) \mathrm{d} t
$$

as was the case in Eq. (2), and the standard deviation of a random step drawn from Eq. (S21) is:

$$
\sqrt{\operatorname{Var}\left(\mathrm{d} \gamma_{t}\right)}=\omega \sqrt{\left(\lambda_{1}+\lambda_{2}+\lambda_{3}\right)}=\omega \sqrt{\frac{2 D_{\gamma} \mathrm{d} t}{\omega^{2}}}=\sqrt{2 D_{\gamma} \mathrm{d} t}
$$

again equal to the standard deviations of increments of Eq. (2). That said, particularly as $\omega$ grows we do not anticipate the stationary distribution of $\gamma$ values arising from Eq. (S21) to be exactly equal to that from Eq. (2). This follows conceptually from the discretisation of $\gamma$ values into multiples of $\omega$ meaning that (for example) the expected value of Eq. (S23) over the distribution of $\gamma$ changes.

We assess the impact of discretised mutation states by using Eq. (S21) to repeat a number of simulations from Figs. 2 and 3 of the main text with differing $\omega$ values. First, in Fig. S12a we plot the standard deviation of $\gamma$ (i.e. $\sigma(\gamma)$ ) for the stationary distribution of cells (with $\gamma_{c}=-\infty$ ) as a function of $\omega$. As $\omega \rightarrow 0$ the impact of discretisation is minimal, (and the solution approaches that for the continuous mutational), whilst for larger $\omega$ we find that $\sigma(\gamma)$ decreases. Following from this we will generally use an intermediate level of discretisation, $\omega=0.5 \phi$ (and thus $\gamma$ can take values $0, \pm 0.5 \phi, \pm \phi, \ldots$ ) for subsequent simulations.

Fig. S12b replicates the results of Fig. 3b of the main text for the discontinuous mutational case. We observe qualitatively similar behaviour, though the phenomena underlying this differ for intermediate $(\alpha \approx 1)$ temporal gradients. For these cases (as illustrated in Fig. S12c) we observe oscillations in population size since each time the rising antibiotic concentration $\left(\gamma_{c}=\alpha t\right)$ passes a multiple of $\omega$ a significant number of 
cells die out (with $\gamma<\gamma_{c}$ ), before the population quickly rebounds towards its maximum carrying capacity. Figs. S12d,e replicate the results of Figs. 3d,e of the main text, again showing close qualitative agreement. In Fig. 3d the discretised mutational states available to our population are apparent, resulting in a step-wise killing curve (discussed in Note S4). Finally, Fig. S12f replicates Fig. 2b of the main text, showing that a similar response to spatial antibiotic gradients is maintained for the discrete mutational case. Our numerical method for quantifying adaptation rate (as described in Note S8) results in the $k=100$ simulation for this stochastic case giving a slightly increased rate of adaptation when compared to the continuous case.
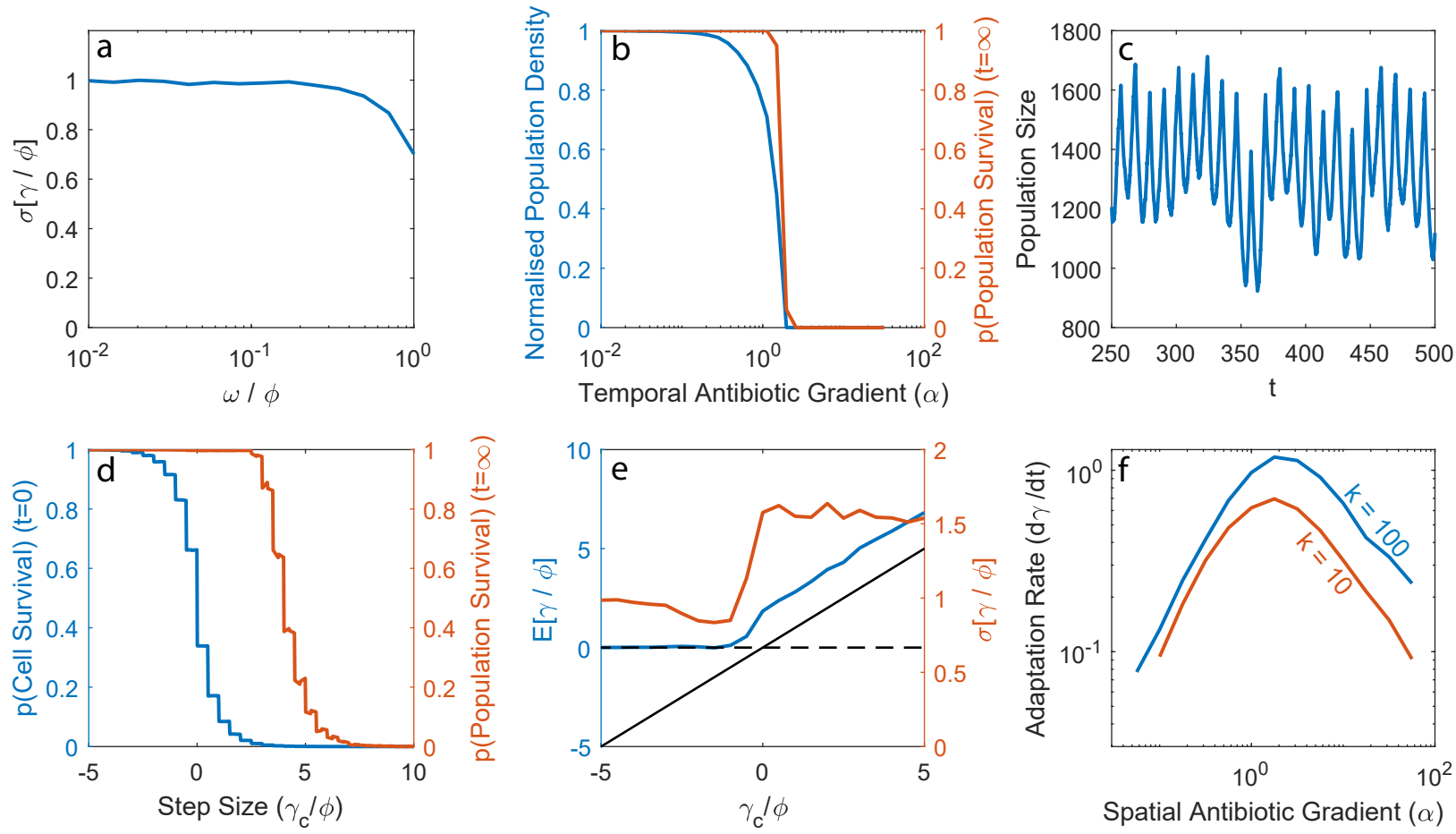

Figure S12: A discontinuous adaptive process a) Standard deviation $(\sigma)$ of the stationary distribution of $\gamma$ values arising from Eq. (S21) when $\gamma_{c}=-\infty$. b) The normalised population density (left axis) and the probability of a population of cells being able to survive indefinitely (right axis) as a function of the temporal gradient in antibiotic concentration (with $\gamma_{c}=\alpha t$ ). This simulation replicates Fig. 3b of the main text (Note S27). c) Mean population size for a population evolving in a temporal antibiotic gradient (as in b) with $\alpha=1$. d) The probability of a single cell surviving (left axis) and the population surviving (right axis) as a function of the size of an antibiotic step rise $\gamma_{c}$ (Note S28). e) The mean $(\mathbb{E}[\gamma / \phi])$ and standard deviation $(\sigma[\gamma / \phi])$ of $\gamma$ for a population of cells allowed to evolve for a long period in the presence of a fixed $\gamma_{c}$ (Note S29). f) The adaptation rate as a function of spatial antibiotic gradient, $\gamma_{c}=\alpha x$, is maximised when $\alpha \approx 1$. Simulation details are presented in Note S30. 


\section{Note S8}

This note accompanies Fig. $2 b$ of the main text.

For each value of $\alpha$ we run a large number (500) of simulations, in each of which a population of $k$ cells starts from $x=0$ at $t=0$, and grows until $t=200$. Initially $\gamma$ values are distributed according to a Laplace distribution with mean 0 and standard deviation $\phi$. After each simulation iteration we record the $\gamma$ value of the cell with the maximal $x$ value. We perform a linear fit to this quantity for the later half of each simulation (i.e. $100<t<200$ ), and the gradient of this fit is $d \gamma / d t$. The value of $d \gamma / d t$ is then averaged over the 500 simulations at each $\alpha$ value.

\section{Note S9}

This note accompanies Fig. 2d of the main text.

For the simulation in Fig. 2d cells evolve on a landscape with a single spatial step in antibiotic concentration from $t=0$ until $t=100$. For $0<x<20$ we have $\gamma_{c}=-\infty$, and for $x>20$ we have $\gamma_{c}=4 \phi$. We initialise each simulation with $20 k$ cells spread from $0<x<20$ with their $\gamma$ values sampled from a Laplace distribution as described in Note S2. The purpose of initialising the simulation with cells spread in $0<x<20$ is to ensure that the time required for diffusion between $x=0$ and $x=20$ is negligible; the only obstacle to overcoming the step is that posed by achieving a sufficiently large $\gamma$ value. We define the step as being "overcome" if there are at least $0.5 \times 10 \times k$ cells with $x>30$ at $t=100$. The purpose of requiring a large number of cells to cross this boundary is to avoid the simulation terminating when only a few cells (with $\gamma<\gamma_{c}$ that cannot replicate in that region) ending it prematurely. We observe that once a small number of cells colonise a large $\gamma_{c}$ region they rapidly grow to maximum density, and so the time taken to go from one cell to near-maximum density in this region is small. We calculate the probability of the step being overcome from the proportion of trials which achieve this within the time limit (1000 trials are performed at each $k$ value).

\section{Note S10}

This note accompanies Fig. S1d of the supplementary material.

This simulation is performed similarly to Note S9 above, but in this case we vary the step size (in Note S9 we had $\gamma_{c}=4 \phi$ ) but keep $k=100$. Each simulation is run until the step is overcome, or $t=1000$ is reached. We can then calculate the probability of a step being overcome within a given timeframe as the proportion of simulations that overcame the step divided by the total number of simulations, in this case 1000 for each step size. As anticipated we observe that if more time is given $(t=1000)$ the probability of overcoming a given step size is significantly increased.

\section{Note S11}

This note accompanies Fig. 2e of the main text.

For this simulation cells evolve on a landscape with two spatial steps in antibiotic concentration from $t=0$ until $t=500$ or the second antibiotic step is overcome. For $0<x<40$ we have $\gamma_{c}=-\infty$, for $40<x<80$ we have variable $\gamma_{c}$, and for $x>80$ we have $\gamma_{c}=6 \phi$. We initialise each simulation with $20 k$ cells spread from $0<x<20$ with their $\gamma$ values sampled from a Laplace distribution as described in Note S2. We define the second step as being overcome when there are $20 \times k$ cells with $x>100$. The time at which this is achieved is $t_{\text {adapt }}$. We calculate the rate of evolution as $t_{\text {adapt }}^{-1}$. For any simulation in which the second step is not overcome before $t=500$ we set this rate to zero. The rate for a given intermediate step 
height is then calculated as the average rate from 1000 trials.

To include a delay we set the time taken to overcome the final step to $t_{2}=t_{\text {adapt }}+1000$ and then use this value to calculate the rate of evolution. We are therefore assuming that the steps are significantly spatially separated such that it takes and additional 1000 time units for the cells to traverse the space between them; this distance is much greater than the inter-step distance actually simulated (40 distance units).

\section{Note S12}

This note accompanies Fig. If of the main text.

For this simulation we simulate a population of cells with a single spatial step similar to Note S9, but here we have $\gamma_{c}=-\infty$ for $x<20$, and for $x>40 \gamma_{c}=2 \phi$. Note that the value of $\phi$ used to define this step is constant across all simulations (it uses the parameters in Table $\mathrm{S} 2$ and so does not depend on $\epsilon$ ). We run 600 trials of each different $\epsilon$ value, and calculate a probability from the fraction of these that overcome the step (defined similarly to Note S9).

To simulate an increased mutation rate we set $\delta=0.1 \times \epsilon$ meaning that an increased value of $\epsilon$ reduces the longevity of cells ( $\delta$ is larger), simulating the impact of increased mutation rate upon their general fitness. We also re-run this simulation with $\delta=1 \times \epsilon$. To reflect the impact of increased mutation rate we also scale Eq. (2) by $\epsilon$, which is equivalent to scaling $\theta$ by $\epsilon$ and $D_{\gamma}$ by $\epsilon^{2}$. This scales the effective value of $\phi$ by a factor of $\epsilon$, but since the step height $\gamma_{c}$ is constant this will impact the probability that it can be overcome: small $\epsilon$ will make it less likely that a fixed step height can be overcome.

Though we have used $\epsilon$ as a simple scaling factor, the means via which a change in mutation rate impacts each parameter may not be as nicely linear as described above. For example, a doubling of mutation rate would not necessarily cause a doubled death rate $(\delta)$; it is probable that $\delta$ would instead be some sub-linear function of $\epsilon$. However, our result (that at both small and large $\epsilon$ the population is unable to overcome a fixed step in antibiotic gradient) will hold quantitatively so long as $\delta$ is a function of $\epsilon$ which satisfies the property that as $\epsilon \rightarrow \infty$ we have $\delta \rightarrow \infty$, and $D_{\gamma} / \theta$ is a function of $\epsilon$ which satisfies the property that as $\epsilon \rightarrow 0$ the variance of $\gamma$ values approaches zero.

\section{Note S13}

This note accompanies Fig. $3 b$ of the main text.

In this figure we simulate a population of cells evolving on an antibiotic concentration ramp defined by $\gamma_{c}(t)=\alpha t$. Each simulation is run from $t=0$ to $t=300$ after which the number of cells alive in the population is recorded. We run 1000 simulations at each $\alpha$ value. The "Normalised Population Density" is the mean final density (over these 1000 simulations) divided by the mean final density when $\alpha=10^{-2}$. This represents the steady-state population density for cells that are not undergoing selection, and when $k=2000$ corresponds to $\approx 1800$ cells. This value is less than the maximum carrying capacity $k$ due to the form of Eq. (S1), which causes the reproduction rate to approach zero as the number of cells competing for resources approaches $k$. The probability that the population survives indefinitely (i.e. as $t \rightarrow \infty$ ) is simply the probability that the steady-state population density is greater than zero. Examples of population die out when $\alpha>1$ are presented in Fig. S2a-f.

\section{Note S14}

This note accompanies Fig. $3 d$ of the main text and Fig. S4 of the supplementary material. 
In this figure we simulate a population of cells for which $\gamma_{c}=-\infty$ when $t<50$ and $\gamma_{c}$ is a constant value for $t>50$. Each simulation is run until $t=400$. The probability that a given cell survives the step increase at $t=50$ is calculated from the number of cells with $\gamma>\gamma_{c}$ at $t=50$. As discussed in Note S4 this result can also be calculated directly from the CDF of the Laplace distribution that arises from Eq. (2). The probability of population survival is the probability that there is at least one cell alive at $t=400$. Essentially every population that has $p($ CellSurvival $)>0$ will survive, and some which have no cells with $\gamma>\gamma_{c}$ may also survive as discussed in the main text and the caption of Fig. S4.

We investigate this later probability by tuning system parameters: In Fig. 3d we plot the probability of population survival when $\theta$ is multiplied by 3 , or $D_{\gamma}$ is divided by 3 . In Fig. S4 we then additionally consider the case when we halve the death rate $(\delta / 2)$ and multiply $\theta$ by 3 . Here we also plot the probability that at least one cell survives the step, which is the probability that any cell has $\gamma>\gamma_{c}$ at $t=50$. The difference between this (the blue) curve and the other curves represents the impact of cells which have $\gamma<\gamma_{c}$ at $t=50$ but evolve to have $\gamma>\gamma_{c}$ before dying. An example of this occurring is presented in Fig. S2g.

\section{Note S15}

This note accompanies Fig. 3e of the main text.

In this simulation we allow a population of $k=1 \times 10^{6}$ cells to evolve for 10,000 time units with a given value of $\gamma_{c}$. We record the mean $\gamma$ value for each population $(\mathbb{E}[\gamma])$ and standard devation of $\gamma(\sigma[\gamma])$ after this time period. Results are then the mean of 10 such trials at each value of $\gamma_{c}$.

\section{Note S16}

This note accompanies Fig. S5a,b of the supplementary material.

In this simulation we allow a population of $k=2000$ cells to evolve for 3,000 time units. Initially $\gamma_{c}=-\infty$, and a step of specified $\gamma_{c}$ is introduced at $t=500$. We record the population size and standard devation of $\gamma(\sigma[\gamma])$ over time for cells that have $\gamma>\gamma_{c}$. The standard deviation is not recorded for all cells because such a metric is perturbed by outlier cells with $\gamma \ll \gamma_{c}$ (which will soon die) immediately following the antibiotic introduction. Results are averaged over 500 such trials at each step size.

\section{Note S17}

This note accompanies Fig. If of the main text.

In this simulation we initialise a population of $k=2000$ of cells at $t=0$ with all cells having a $\gamma$ value of zero. We initially set $\gamma_{c}=-\infty$ or 0 . Cells are allowed to proliferate for a varying amount of time, $t_{p}$, following which we set $\gamma_{c}=2.5 \phi$. We then run the simulation for a further 200 time units, after which we record the number of cells alive in the system: If there are live cells at this point then the population has survived. We repeat 1000 trials at each $t_{p}$ value in order to calculate a probability of population survival.

\section{Note S18}

This note accompanies Fig. S6a,b of the supplementary material.

In this simulation we initialise a population of cells at $t=0$ with a mean $\gamma$ value of $10 \phi$. For $0<t<200$ we have $\gamma_{c}=10 \phi$, for $200<t<200+t_{R}$ we have $\gamma_{c}=-\infty$, and for $200+t_{R}<t<400+t_{R}$ we have $\gamma_{c}=10 \phi$. The simulation is then stopped when $t=400+t_{R}$. The probability of population survival (Fig. S6a) is the probability that there is at least one cell alive at $t=400+t_{R}$, whereas the probability of cell 
survival (Fig. S6b) is the proportion of cells that have $\gamma>10 \phi=\gamma_{c}$ when $t=200+t_{R}$. Results are averaged over 1000 simulation trials at each $t_{R}$.

\section{Note S19}

This note accompanies Fig. S6c,d of the supplementary material.

In this simulation we initialise a population of cells at $t=0$ with a mean $\gamma$ value of 0 . For $0<t<t_{A}$ we have $\gamma_{c}=10 \phi$, and then for $t_{A}<t<200+t_{A}$ we have $\gamma_{c}=-\infty$. The simulation is then stopped when $t=200+t_{A}$. The probability of population survival (Fig. S6c) is the probability that there is at least one cell alive at $t=200+t_{A}$, whereas the probability of cell survival (Fig. S6d) is the proportion of cells that are still alive when $t=t_{A}$. Results are averaged over 1000 simulation trials at each $t_{A}$.

\section{Note S20}

This note accompanies Fig. S6e of the supplementary material.

In this simulation we initialise a population $k=2000$ of cells at $t=0$ with a mean $\gamma$ value of zero. We initially set $\gamma_{c}=3.5 \phi$, meaning that most of the initial cells will have $\gamma<\gamma_{c}$ at $t=0$ and will consequently die out. Cells are allowed to adapt to $\gamma_{c}=3.5 \phi$ for a varying amount of time, $t_{\text {adapt }}$, following which we set $\gamma_{c}=7 \phi$. We then run the simulation for a further 400 time units, after which we record the number of cells alive in the system: If there are live cells at this point then the population has survived. We repeat 1000 trials at each intermediate step time in order to calculate a probability of population survival.

\section{Note S21}

This note accompanies Fig. 4a-c of the main text. and Fig. S7a-c of the supplementary material.

We simulate two competing strategies with identical parameter values (as in Note S3) that differ in two cases: In case $1 \theta_{1}=\theta / \sqrt{1-\zeta}$ and $\theta_{2}=\theta / \sqrt{\zeta}$, and $D_{\gamma_{1}}=D_{\gamma_{2}}=D_{\gamma}$. In case $2 D_{\gamma_{1}}=D_{\gamma} \sqrt{1-\xi}$ and $D_{\gamma_{2}}=D_{\gamma} \sqrt{\xi}$, and $\theta_{1}=\theta_{2}=\theta$. In both cases $D_{\gamma}$ and $\theta$ remain equal to their values in Table S2. We select these relations such that the initial distribution of $\gamma$ values for the combined strategy $\left(\gamma_{1}+\gamma_{2}\right)$ is identical to the distribution if just one strategy was present. Both strategies are initialised using Laplace distributions with mean $\gamma$ of zero and standard deviations $\phi_{1}\left(D_{\gamma_{1}}, \theta_{1}\right)$ and $\phi_{2}\left(D_{\gamma_{2}}, \theta_{2}\right)$. They then evolve on a temporal ramp in antibiotic of gradient $\alpha=1.2$ (with $\gamma_{c}(t)=\alpha t$ ) for 1000 time units. We record the population's mean $\gamma_{1}$ and $\gamma_{2}$ values at each iteration. From this we calculate the adaptation rate $(d \gamma / d t)$ for each strategy and their combination $(\bar{\gamma})$ (removing data for simulation runs during which the population dies out). The proportion of simulations (each of length 1000 time units) during which all cells die out is used to calculate the probability of population survival in Fig. 4a. Each simulation in Fig. 4a-c represents statistics generated from the outcome of 1000 trials at each $\zeta$ or $\xi$ value.

\section{Note S22}

This note accompanies Fig. $4 d$ of the main text and Figs. S7d-e of the supplementary material.

This simulation is similar to that described in Note S21, but we introduce an antibiotic step of height $3 \phi$ (where $\phi$ is calculated using the parameters in Table S2, independently of $\zeta$ and $\xi$ ) at $t=0$. Each simulation is run for a long time (to $t=20,000$ ), after which we record which strategy has "won" (defined as that with the greater mean $\gamma_{i}$ value across the population). In Figs. S7d-e we plot the quantity $\mathbb{E}\left[\left|\gamma_{1}-\gamma_{2}\right| /\left(\gamma_{1}+\gamma_{2}\right)\right]$, using the final population mean values of $\gamma_{1}$ and $\gamma_{2}$. Results are averaged over 100 simulation trials at each $\zeta$ or $\xi$ value. 


\section{Note S23}

This note accompanies Figs. S7f,g of the supplementary material.

These simulations are similar to those described in Note S22, but we initialise Strategy 1 with a mean $\gamma_{1}$ value of $4 \phi$. We are thus aiming to assess whether it is possible for a second strategy (in this case $\gamma_{2}$ ) to overcome $\gamma_{1}$ 's initial advantage. Results are averaged over 100 simulation trials at each $\zeta$ or $\xi$ value.

\section{Note S24}

This note accompanies Figs. S7h,i of the supplementary material.

These simulations are similar to case 1 described in Note S21, but we set $\theta_{1}=\theta$ and $\theta_{2}=\eta \theta$. Each simulation represents statistics generated from the outcome of 500 trials at each $\eta$ value.

\section{Note S25}

This note accompanies Figs. 4e,f of the main text.

This simulation is similar to that in Note S21, but now we consider a spatial ramp with $\alpha=1$ (so $\gamma_{c}(x)=\alpha x$ ). All parameters and definitions remain the same, aside from the differences (in terms of $k$ and $\sigma$ ) defined in Table S2. We calculate the adaptation rate as described in Note S8 for each strategy, with each simulation being run for 200 time units. Results are averaged over 1000 simulation trials at each $\zeta$ or $\xi$ value.

\section{Note S26}

This note accompanies Fig. S8 of the supplementary material.

In this simulation we consider a spatial step in antibiotic concentration where for $0<x<20$ we have $\gamma_{c}=-\infty$, and for $x>20$ we have $\gamma_{c}=4 \phi_{0}$ (with $\phi_{0}$ defined as in Note S22). At the end of each simulation we calculate the mean $\gamma_{i}$ values for cells with $x>30$. These are then used to quantify whether a given strategy has "Won" in overcoming the step (in this case if the population's mean $\gamma_{1}$ is greater than its mean $\left.\gamma_{2}\right)$, as well as the quantity $\mathbb{E}\left[\left|\gamma_{1}-\gamma_{2}\right| /\left(\gamma_{1}+\gamma_{2}\right)\right]$. Each simulation is run for 200 time units, and results are averaged over 500 trials.

\section{Note S27}

This note accompanies Fig. S12b,c of the supplementary material.

This simulation is largely the same as that described in Note S13. We set $\omega=0.5 \phi$. As before we initialise the simulation with a Laplace distribution of $\gamma$, and then we round each $\gamma$ value to the nearest multiple of $\omega$. We run 100 trials of length 500 time units at each $\alpha$ value. We define the simulation output in each case as the mean population size over the time period $t \in[250,500]$. This allows the effect of oscillations (as observed in Fig. S12c due to discretisation of $\gamma$ ) to be minimised.

\section{Note S28}

This note accompanies Fig. S12d of the supplementary material. 
This simulation is largely the same as that described in Note S14. As in Note S27 we set $\omega=0.5 \phi$, and discretise the initial Laplace distribution of $\gamma$ at the start of the simulation. We perform 1,500 trials at each step size.

\section{Note S29}

This note accompanies Fig. S12e of the supplementary material.

This simulation is largely the same as that described in Note S15. As in Note S27 we set $\omega=0.5 \phi$, and discretise the initial Laplace distribution of $\gamma$ at the start of the simulation. We initialise a population of

$10^{5}$ cells and allow them to evolve for 10,000 time units. Results are then the mean of 10 such trials at each value of $\gamma_{c}$.

\section{Note S30}

This note accompanies Fig. S12f of the supplementary material.

This simulation is largely the same as that described in Note S8. As in Note S27 we set $\omega=0.5 \phi$, and discretise the initial Laplace distribution of $\gamma$ at the start of the simulation. 


\section{Supplementary References}

[1] R. Hermsen, "The adaptation rate of a quantitative trait in an environmental gradient," Physical Biology, vol. 13, no. 6, 2016.

[2] D. T. Gillespie, "Approximate accelerated stochastic simulation of chemically reacting systems," Journal of Chemical Physics, vol. 115, no. 4, pp. 1716-1733, 2001.

[3] Y. Q. Liu, Y. Z. Zhang, and P. J. Gao, "Novel Concentration-Killing Curve Method for Estimation of Bactericidal Potency of Antibiotics in an In Vitro Dynamic Model," Antimicrobial Agents and Chemotherapy, vol. 48, no. 10, pp. 3884-3891, 2004.

[4] J. C. Fu and T.-L. Wu, "Linear and nonlinear boundary crossing probabilities for brownian motion and related processes," Journal of Applied Probability, vol. 47, pp. 1058-1071, 2010.

[5] H. Robbins and D. Siegmund, "Boundary Crossing Probabilities for the Wiener Process and Sample Sums," The Annals of Mathematical Statistics, vol. 41, no. 5, pp. 1410-1429, 1970.

[6] E. Toprak, A. Veres, J. B. Michel, R. Chait, D. L. Hartl, and R. Kishony, "Evolutionary paths to antibiotic resistance under dynamically sustained drug selection," Nature Genetics, vol. 44, no. 1, pp. 101-105, 2012.

[7] G. Chevereau, M. Dravecká, T. Batur, A. Guvenek, D. H. Ayhan, E. Toprak, and T. Bollenbach, "Quantifying the Determinants of Evolutionary Dynamics Leading to Drug Resistance," PLoS Biology, vol. 13, no. 11, pp. 1-18, 2015.

[8] J. E. Barrick and R. E. Lenski, "Genome dynamics during experimental evolution," Nature Reviews Genetics, vol. 14, no. 12, pp. 827-839, 2013.

[9] R. F. Bass, "Stochastic differential equations with jumps," Probability Surveys, vol. 1, pp. 1-19, 2004. 